\title{
An Easy-to-Understand Method to Construct Desired Distance-Like Measures
}

\author{
Wen Qing Fu $\mathbb{D}^{1,2}$, Sheng Gang Li $\mathbb{D}^{1},{ }^{1}$ Harish Garg $\mathbb{D}^{3}$ Heng Liu ${ }^{3}{ }^{4}$ \\ Ahmed Mostafa Khalil $\mathbb{D}^{5}{ }^{5}$ and Jingjing Zhao ${ }^{6}{ }^{6}$ \\ ${ }^{1}$ College of Mathematics and Statistics, Shaanxi Normal University, Xi'an 710062, China \\ ${ }^{2}$ Freshman Academy, Xi'an Technological University, Xi'an 710021, China \\ ${ }^{3}$ School of Mathematics, Thapar Institute of Engineering \& Technology, Deemed University, Patiala 147004, Punjab, India \\ ${ }^{4}$ School of Science, Guangxi University of Nationalities, Nanning 530006, China \\ ${ }^{5}$ Department of Mathematics, Faculty of Science, Al-Azhar University, Assiut 71524, Egypt \\ ${ }^{6}$ School of Psychology, Shaanxi Normal University, Xi'an 710062, China
}

Correspondence should be addressed to Sheng Gang Li; shengganglinew@126.com and Harish Garg; harishg58iitr@gmail.com

Received 5 February 2021; Accepted 10 June 2021; Published 8 July 2021

Academic Editor: Wei Wang

Copyright (C) 2021 Wen Qing Fu et al. This is an open access article distributed under the Creative Commons Attribution License, which permits unrestricted use, distribution, and reproduction in any medium, provided the original work is properly cited.

\begin{abstract}
Metrics and their weaker forms are used to measure the difference between two data (or other things). There are many metrics that are available but not desired by a practitioner. This paper recommends in a plausible reasoning manner an easy-to-understand method to construct desired distance-like measures: to fuse easy-to-obtain (or easy to be coined by practitioners) pseudo-semimetrics, pseudo-metrics, or metrics by making full use of well-known t-norms, t-conorms, aggregation operators, and similar operators (easy to be coined by practitioners). The simple reason to do this is that data for a real world problem are sometimes from multiagents. A distance-like notion, called weak interval-valued pseudo-metrics (briefly, WIVP-metrics), is defined by using known notions of pseudo-semi-metrics, pseudo-metrics, and metrics; this notion is topologically good and shows precision, flexibility, and compatibility than single pseudo-semi-metrics, pseudo-metrics, or metrics. Propositions and detailed examples are given to illustrate how to fabricate (including using what "material") an expected or demanded WIVP-metric (even intervalvalued metric) in practical problems, and WIVP-metric and its special cases are characterized by using axioms. Moreover, some WIVP-metrics pertinent to quantitative logic theory or interval-valued fuzzy graphs are constructed, and fixed point theorems and common fixed point theorems in weak interval-valued metric spaces are also presented. Topics and strategies for further study are also put forward concretely and clearly.
\end{abstract}

\section{Introduction and Preliminaries}

In many cases, the measure values of true data are not unique (but two or more) for uncertainty or complexity. For example, there are several agents in China who value and order all periodicals published in China. Peking University Library and Nanjing University Library are generally thought to be the best two and incomparable to each other. For a journal J, assume that the orders given by Peking University Library and the Nanjing University Library are $m$-th and $n$-th, respectively; then, $m$ and $n$ may be not the same in general. There are also many other examples. In 2012, breakthrough selected by the famous journal Science are different from those selected by the famous journal Nature; Gini coefficients in China in 2012 from two different agents are 0.481 and 0.61 , respectively; the Chebyshev distance (resp., the Euclidean distance, the Manhattan distance or the city block distance, and the river distance) between two points $(0,1)$ and $(1,2)$ in the Euclidean plane $R^{2}$ is 1 (resp., $\sqrt{2}, 2,3$ ). Please see Proposition 1 for definitions of these metrics; the effective distances used in cluster analysis are many and varied; a given asymptomatic infected people to Corona Virus Disease (COVID-19 for short) are thought to be highly contagious (which can be represented by a fuzzy number $A$ ) 
by experts in one country but lowly contagious (which can also be represented by a fuzzy number $B$ that is much different from $A$ ) by experts in another country.

In practice, most people choose just one of the measure values (or choose the arithmetic mean of these measure values) as the true data; such a kind of dispose can be accepted only in rare cases (e.g., the information loss cannot be avoided or make almost no difference). To make an improvement in the disposal of these uncertain or complex data, at least two better theories (One is theoretically inspirational, another is application-motivated; both are based mainly on the idea of fuzzy set.) have been proposed which are mostly about measuring values of difference between two abstract "points" (precisely, two elements of a set) whose information or data can be provided by at least two different agents (but cannot be provided satisfactorily by one agent, see Example 1).

\section{Example 1}

(1) The distance between two fuzzy sets $A=\{(0.1 / x),(0.3 / y)\}$ and $B=\{(0.6 / u),(0.8 / v)\}$ cannot be expressed accurately (or satisfactorily) in a real number but can be expressed satisfactorily in a 4-element set $H=\{|0.1-0.6|,|0.1-0.8|,|0.3-0.6|, \mid 0.3$ $-0.8 \mid\}=\{0.3,0.5,0.7\}$, or an interval number $d=\langle 0.3,0.7\rangle$. $d$ can be determined by two elements (even if it is an infinite set). Therefore, all sets involved here can be thought to be finite.

(2) The distance between two bodies $A=\{(x, y$, $\left.z) \in R^{3} \mid x^{2}+y^{2}+z^{2} \leq \epsilon^{2}\right\}$ (i.e., the closed ball in the 3dimensional Euclidean space $R^{3}$ with center $(0,0,0)=$ $\overrightarrow{0}$ and radius $\left.\epsilon^{2}\right)$ and $B=\{(x, y, z) \epsilon$ $\left.R^{3} \mid\left(x-\epsilon^{2}-\varepsilon\right)^{2}+y^{2}+z^{2} \leq \varepsilon^{2}\right\}$ (i.e., the ball in $R^{3}$ with center $(\epsilon+\varepsilon, 0,0)$ and radius $\epsilon)$ cannot be expressed accurately (or satisfactorily) in a real number but can be expressed satisfactorily in several numbers (where $0 \leq \epsilon \leq \varepsilon)$. For instance, it can be expressed in a 3-element set $\{a, \dot{a}, \ddot{a}\}$ (in this way information loss can be almost avoided) or be expressed by any 2-element subset of $\{a, \dot{a}, \ddot{a}\}$ (in this way much more information loss can be avoided), where $a=0=\inf \left\{\rho_{2}(\vec{x}, \vec{y})\right.$ $\mid \vec{x} \in A, \vec{y} \in B\}\left(\rho_{2}\right.$ is the ordinary Euclidean metric on $\left.R^{3}\right), \dot{a}=\epsilon+\varepsilon$ is the Euclidean distance between the centroid $(0,0,0)=\overrightarrow{0}$ of $A$ and the centroid $(\epsilon+\varepsilon, 0,0)$ of $B$, and $\ddot{a}=2 \varepsilon$ is the Hausdorff distance between $A$ and $B$ (notice that $a \leq \dot{a} \leq \ddot{a}$ ).

(3) During the time of COVID-19 (especially, the first three months), a lot of things (particularly, traveling and meeting) had to stop in China. The expected College back-to-University time can be forecasted rather than accurately based on several (even the first two of them) time series (the time series of suspected cases, the time series of imported cases, the time series of close contact cases, the time series of no symptoms cases, the time series of cluster infection cases, the time series of confirmed cases, etc.) but cannot be forecasted satisfactorily by one of them.
The first way to make an improvement is using a fuzzy metric. Several authors have introduced the concepts of fuzzy metric and fuzzy metric space from different points of view [1-4]. Erceg [1] defined a fuzzy pseudo-metric on a set consisting of fuzzy sets (e.g., fuzzifications of data or information to be disposed) by generalizing the Hausdorff distance between usual sets. The motivation behind the fuzzy metric of Kramosil and Michalek [3] was a statistical metric; recently, these kinds of fuzzy metric spaces have stimulated a lot of interest (see [5] and references herein for details). Kaleva and Seikkala's metric [2] defined the distance between two points as a fuzzy number for the reason that sometimes uncertainty is due to fuzziness rather than randomness. As is known, Kaleva and Seikkala's fuzzy metric spaces possess rich structures with suitable choices of binary operations. Much theoretical work related to Kaleva and Seikkala's fuzzy metric spaces has been done in recent years (see [2, 5-9] and references herein). By presenting intuitive level forms for the triangle inequalities in the definition of Kaleva and Seikkala's fuzzy metric, Huang and $\mathrm{Wu}$ [6] offered a new and convenient tool for the description and analysis of fuzzy metric spaces (cf. their subsequent work [7] on the existence and uniqueness of completion of special fuzzy metric spaces). Under some intuitive and mild assumptions, Fang [5] (as an improvement on Huang and $\mathrm{Wu}$ [7]) also proved the existence and the uniqueness of completion of fuzzy metric spaces. Xiao et al. [10] took a closer step toward possible applications of fuzzy metrics (in integral equations, differential equations, qualitative behavior, dynamic systems, and other nonlinear problems), in which the authors studied the existence and uniqueness of fixed points (under a weaker assumption) for nonlinear contractions in fuzzy metrics spaces in the sense of Kaleva and Seikkala [2]. By virtue of a level-cut method, they established relationships between a fuzzy metric and a family of quasi-metrics (so that the utilizing of results and skills in metric spaces becomes more and more possible).

The second way is using a dissimilarity measure or a distance measure, which is defined on a set consisting of special fuzzy sets (e.g., fuzzifications of data or information to be disposed); it takes many steps toward practical applications of metrics. Balopoulos et al. [11] defined a new family of distance measures (based on matrix norms) for binary operators on $[0,1]$, which can also be used to measure the difference between two fuzzy sets on two finite sets. Bustince et al. [12] constructed distance measures, proximity measures, and fuzzy entropies by aggregating restricted dissimilarity functions in a special way. Liu [13] gave systematically an axiom definition of entropy, distance measure, and similarity measure of fuzzy sets; he also discussed basic relations between these measures. Fan et al. [14] defined a new divergence measure, study the relations between fuzzy entropy, fuzzy Hamming distance, and divergence measures defined. They obtain quite a general conclusion and solve a problem proposed by Liu [13]. Dissimilarity measure and distance measure have already found wide applications for their intuitiveness (see [15-21]).

As far as data are concerned, fuzzy metrics mentioned above have some shortcomings. It seems not very convenient 
to apply these fuzzy metrics in practical problems because axioms satisfied by these metrics are complex; implementation of computing related to these fuzzy metric spaces is obviously difficult because operations (even if addition or subtraction) or sorting of fuzzy numbers cannot be realized in computers unless the fuzzy numbers involved are extremely simple (for example, they are triangle fuzzy numbers or interval numbers). Dissimilarity measures and distance measures mentioned above also have some faults. For example, they have few nice topological properties (see $[8,11-13,16-21])$ because they do not satisfy the triangle inequality in general; axioms they satisfy are also complex. These naturally urge people to find a kind of metrics (or their weaker forms) which are down to earth, i.e., they are theoretically good, intuitive (because intuition and examples always provide inspirations for both theoretical study itself and its applications in the real world), and easyto-use. For example, try to find a metric (or its weaker form) such that the complexity of the distance value of two points is between that of a real number and that of a fuzzy number.

An immediately thought way to construct such a metric (or its weaker form) $\rho$ is to take it as a weighted mean of some well-known or usually used metrics $\rho_{1}, \ldots, \rho_{n}$, i.e., let $\rho=k_{1} \rho_{1}+\cdots+k_{n} \rho_{n}$, where $k_{1}, \ldots, k_{n} \in(0,1)$ satisfies $k_{1}+\cdots+k_{n}=1$. However, information lost in this way may be too much compared to a way based on interval numbers (unless the set $\left\{k_{1}, \ldots, k_{n}\right\}$ is chosen as the optimal one such that $\rho(x, y)$ is the fittest value for each $\left.(x, y) \in X^{2}\right)$.

Interval numbers or interval data appear in many cases (such as numerical analysis-handling rounding errors, computer-assisted proofs, global optimization, particularly, modeling uncertainty) because the data involved there cannot be accurately expressed in real numbers but can be expressed in interval numbers. The measure values of true data can be looked as an interval data, i.e., one can use an interval number to denote the measure values of true data. For example, the distance between $A$ and $B$ in Example 1 (2) can be expressed as one of the following six interval numbers: $[a, a]$ (or $\langle a, a\rangle),[\dot{a}, \dot{a}]$ (or $\langle\dot{a}, \dot{a}\rangle),[\ddot{a}, \ddot{a}]$ (or $\langle\ddot{a}$ , $\ddot{a}\rangle),[a, \dot{a}]$ (or $\langle a, \dot{a}\rangle),[a, \ddot{a}]$ (or $\langle a, \ddot{a}\rangle),[\dot{a}, \ddot{a}]$ (or $\langle\dot{a}, \ddot{a}$ $>)$.

Motivated by Polya's plausible reasoning [22], the present paper will consider a fusion of easy-to-obtain measure data (e.g., values of various easy-to-obtain pseudosemi-metrics, pseudo-metrics, or metrics) by making full use of well-known t-norms, t-conorms, aggregation operators, and similar operators coined by practitioners; of course, interval numbers and triangular fuzzy numbers can also be used. In Section 2, a distance-like notion, called weak interval-valued pseudo-metrics (briefly, WIVP-metrics), is defined by using known notions of pseudo-semi-metrics, pseudo-metrics, and metrics (this notion and its special cases are also characterized by using axioms and connections between these notions and other known and related notions); propositions and detailed examples are given to illustrate how to fabricate (including using what "material") an expected or demanded WIVP-metric (even intervalvalued metric) in practical problems. Sections 3 and 4 are on theoretical applications of WIVP-metrics, where we demonstrate how to construct, by using some logic implication operators, some WIVP-metrics which may be useful in quantitative logic (cf. [23]) and quantitative reasoning (cf. [24]), and how to define well matched interval-valued metrics on interval-valued fuzzy graphs. Fixed point theorems and common fixed point theorems in WIVP-metrics are presented in Section 5. We end the paper with concluding remarks in Section 6.

Now, we present some preliminary notions needed in this paper.

Let $[0,1]^{R}$ be the set of all fuzzy sets on $R$ (i.e., the set of all mappings from $R$ to $[0,1]$ ), where $R$ is the set of all real numbers (also called the real line) and $[0,1]$ is the closed unit interval of $R$. For each subset $Y \subseteq R$ and each $b \in[0,1]$, we use $b_{Y}$ to denote the fuzzy set on $R$ taking value $b$ on $Y$ and 0 elsewhere; we will make no distinction between $1_{Y}$ and $Y$. If a fuzzy set $\mu \in[0,1]^{R}$ satisfies $\mu(r)=1$ for some $r \in R$ and $\left[\mu_{a}{ }^{-}, \mu_{a}{ }^{+}\right]$(i.e., a closed interval of $R$ ) for any $a \in(0,1]$, then we call $\mu$ a fuzzy number (we will regard $\mu-$ and $\mu+$ as functions on $(0,1]$, cf. $[14,24,25])$. The set of all fuzzy numbers is denoted by $\mathscr{F}$. A fuzzy number $\mu$ is called nonnegative if supp $\mu \subseteq[0,+\infty$ ) (where supp $\mu=\{x$ $\epsilon R \mid \mu(x)>0\}$ is called the support of $\mu$ ). The set of all nonnegative fuzzy numbers is denoted by $\mathscr{F}+$. Fuzzy numbers are the most commonly used fuzzy sets on the real line (see [25-30] and references herein); one of their applications is that they can be used to define fuzzy metric space (an important notion in fuzzy analysis). A binary operation * on $[0,1]$, which is commutative (or symmetric), associative, and nondecreasing coordinately, is said to be a $\mathrm{t}$-norm on $[0,1]$ if it satisfies $a * 1=a(\forall a \in[0,1]) ;{ }^{*}$ is said to be a t-conorm on $[0,1]$ if it satisfies $a * 0=a(\forall a$ $\in[0,1])$.

Definition 1 (see [2]). A fuzzy metric space is a quadruple $(X, d, L, R)$ (in this case, $d: X \times X \longrightarrow \mathscr{F}+$ is called a fuzzy metric) which satisfies the following two conditions ( $\forall(x$, $\left.y, z) \in X^{3}\right)$ :

(1) $d$ is positive, i.e., $\left(d(x, y)=0_{R} \Leftrightarrow x=y\right)$ and symmetric, (i.e., $d(x, y)=d(y, x)$ )

(2)

(i) if $s \leq \lambda_{1}(x, z), t \leq \lambda_{1}(z, y)$, and $s+t \leq \lambda_{1}(x, y)$, then $d(x, y)(s+t) \geq L(d(x, z)(s), d(z, y)(t))$

(ii) if $s \geq \lambda_{1}(x, z), t \geq \lambda_{1}(z, y)$, and $s+t \geq \lambda_{1}(x, y)$, then $d(x, y)(s+t) \leq R(d(x, z)(s), d(z, y)(t))$

Here, $d: X \times X \longrightarrow \mathscr{F}+$ is a mapping, $L$ and $R$ are operations on $[0,1]$ which are symmetric, nondecreasing coordinately and satisfy $L(0,0)=0$ and $R(1,1)=1$, and $d(x$, $y)_{[a]}=\left[\lambda_{a}(x, y), v_{a}(x, y)\right](\forall x, y \in X, \forall a \in(0,1])$.

Definition 2 (see [31]). An interval number (i.e., a special kind of fuzzy number) is a point $\mathbf{a}=\langle a-, a+\rangle$ in the 2dimensional Euclidean space $R^{2}$ which satisfies $a-\leq a+$. The set of all interval numbers (with the point-wise order $\leq$ ) is denoted by $\square(R)$ (Notice that a total order $[32,33] \leq$ was also defined on $\square(R):\langle a-, a+\rangle \leq\langle b-, b+\rangle \Leftrightarrow 0.5(a-+a$ 
$+)<0.5(b-+b+)$ or $0.5(a-+a+)=0.5(b-+b+)$ but $a$ $+-a-\geq b+-b-$. Moreover, we will identify a closed interval $[a, b]$ of $R$, a point $\langle a, b\rangle$ in $R^{2}$, and a fuzzy number $\left.1_{[a}, b\right]$ since there exists a natural one-to-one correspondence between the set of all closed intervals of $R$ and $\square(R)$.). For any $\langle a-, a+\rangle,\langle b-, b+\rangle \in \llbracket(R)$ and each nonnegative real number $r$, define $\langle a-, a+\rangle \oplus\langle b-, b+\rangle=\langle a-+b-, a$ $++b+\rangle,\langle a-, a+\rangle \ominus\langle b-, b+\rangle=\langle\min \{a--b-, a+-b$ $+\}, \max \{a--b-, a+-b+\}\rangle$, and $r\langle a-, a+\rangle=\langle r a-, r a$ $+\rangle$. When $a-\rangle b-$ and $a+\rangle b+$, write $\langle a-, a+\rangle \gg\langle b$ $-, b+\rangle$ (or $\langle b-, b+\rangle \ll\langle a-, a+\rangle)$; when $a-=a+=a$, write $\langle a-, a+\rangle=a$. For every subinterval $J \subseteq R$, write $\llbracket(J)$ $=\{\langle a-, a+\rangle \in \mathbb{Q}(R) \mid a-, a+\in J\}$.

\section{Definition and Examples of WIVP-Metric}

In this section, we will define the notion of weak intervalvalued pseudo-metrics (shortly, WIVP-metrics), exemplify in detail how to construct distance-like measures (including WIVP-metrics) desired in practice by fusing easy-to-obtain or easy-to-coin pseudo-semi-metrics, pseudo-metrics, or metrics based on operators $\wedge, \vee$, and simple aggregation operators. We also characterize WIVP-metric and its special forms intuitively so that practitioners can understand them easily.

Definition 3. Let $X$ be a set, $\rho: X \times X \longrightarrow \square(R+)$ a mapping $($ where $R+=[0,+\infty))$. If $\rho-=p_{1} \circ \rho: X \times X \rightarrow[0$, $+\infty$ ) is a pseudo-semi-metric ( $\rho-$ is called a pseudo-semimetric on $X$ if it satisfies $\rho-(x, x)=0(\forall x \in X)$ and $\rho-(x$, $y)=\rho-(y, x)(\forall(x, y) \in X \times X) ; \rho-$ is called a pseudometric on $X$ if it is a pseudo-semi-metric on $X$ satisfying triangle inequality; $\rho$ - is called a semi-metric on $X$ if it is a pseudo-semi-metric on $X$ satisfying $\{((x, y) \in X \times X) \mid \rho$ $-(x, y)=0\}=\Delta(X)$, where $\Delta(X)=\{(x, x) \mid x \in X\}$ is the diagonal of $X^{2}$.) (resp., a pseudo-metric, a semi-metric, a metric) on $X$ and $\rho+=p_{2} \circ \rho: X \times X \longrightarrow[0,+\infty)$ is a pseudo-metric (resp., a pseudo-metric, a metric, a metric) on $X$, then $\rho$ is called a WIVP-metric on $X$ (resp., an intervalvalued pseudo-metric on $X$, a weak interval-valued metric on $X$, an interval-valued metric on $X)$ and $(X, \rho)$ is called a WIVP-metric space (resp., an interval-valued pseudo-metric space, a weak interval-valued metric space, an intervalvalued metric space), where $p_{1}: R^{2} \longrightarrow R$ and $p_{2}$ $: R^{2} \longrightarrow R$ are the first coordinate projection and the second coordinate projection, respectively (For a WIVP-metric on $X$, write $\widehat{\mathscr{B}}_{1}=\{\widehat{B}(x, \mathbf{a}) \mid x \in X, a \in(0,+\infty)\}, \widehat{\mathscr{B}}_{2}=\{\widehat{B}(x$, $\langle a-, a+\rangle) \mid x \in X,\langle a-, a+\rangle \in \mathbb{Q}(R),\langle a-, a+\rangle \gg 0\}$, $\widehat{\mathscr{B}}_{\rho}(x)=\{\widehat{B}(x,\langle a-, a+\rangle) \mid\langle a-, a+\rangle \in \mathbb{Q}(R),\langle a-, a+\rangle$ $\gg 0\}(x \in X), \widehat{\mathscr{T}}_{1}, \rho=\left\{\bigcup \mathscr{A} \mid \mathscr{A} \subseteq \widehat{\mathscr{B}}_{1}\right\}, \widehat{\mathscr{T}}_{2}, \rho=\{\bigcup \mathscr{A} \mid \mathscr{A}$ $\left.\subseteq \widehat{\mathscr{B}}_{2}\right\}, \widehat{\mathscr{T}}_{\rho}=\left\{W \in 2^{X} \mid \widehat{B}(x,\langle a-, a+\rangle) \subseteq W\right.$ for some $\langle a-$, $a+\rangle \gg 0$ whenever $x \in W\}$, where $\widehat{B}(x,\langle a-, a+\rangle)=$ $\{y \in X \mid \rho(x, y) \geq\langle a-, a+\rangle\}$. Then, $\widehat{\mathscr{T}}_{\rho}=\left\{W \in 2^{X} \mid \widehat{B}(x, \mathbf{a})\right.$ $\subseteq W$ for some a $\gg 0$ whenever $x \in W\} \subseteq \widehat{\mathscr{T}}_{1}, \rho \subseteq \widehat{\mathscr{T}}_{2,} \rho, \widehat{\mathscr{T}}_{\rho}$ is a topology on $X$, and both $\widehat{\mathscr{T}}_{1} \rho$ and $\widehat{\mathscr{T}}_{2,} \rho$ are pre-topologies on $X$. It can be proved that $\widehat{\mathscr{T}}_{\rho}, \widehat{\mathscr{T}}_{1, \rho}$, and $\widehat{\mathscr{T}}_{2,} \rho$ have good topological properties.).
Proposition 1. Let $R^{3}$ be the 3-dimension Euclidean space and $\rho_{1}, \rho_{2}, \rho_{3}$, and $\rho_{4}$ be the Chebyshev metric, the Euclidean metric, the Manhattan metric or the city block metric, and the river metric on $R^{3}$, respectively (We will consider them to be metrics on $R^{2}$ by identifying a point $\left(a_{1}, a_{2}\right) \in R^{2}$ with a point $\left(a_{1}, \underline{a_{3}}, 0\right) \in R^{3}$.), defined by (where $\vec{a}=\left(a_{1}, a_{2}, a_{3}\right) \in R^{3}$ and $\left.\vec{b}=\left(b_{1}, b_{2}, b_{3}\right) \in R^{3}\right)$,

$$
\begin{aligned}
& \rho_{1}(\vec{a}, \vec{b})=\max \left\{\left|a_{1}-b_{1}\right|,\left|a_{2}-b_{2}\right|,\left|a_{3}-b_{3}\right|\right\}, \\
& \rho_{2}(\vec{a}, \vec{b})=\sqrt{\left(a_{1}-b_{1}\right)^{2}+\left(a_{2}-b_{2}\right)^{2}+\left(a_{3}-b_{3}\right)^{2},} \\
& \rho_{3}(\vec{a}, \vec{b})=\left|a_{1}-b_{1}\right|+\left|a_{2}-b_{2}\right|+\left|a_{3}-b_{3}\right|, \\
& \rho_{4}(\vec{a}, \vec{b})= \begin{cases}\rho_{2}(\vec{a}, \vec{b}), & a_{1}=b_{1}, \\
\sqrt{a_{2}^{2}+a_{3}^{2}}+\sqrt{b_{2}^{2}+b_{3}^{2}}+\left|a_{1}-b_{1}\right|, & a_{1} \neq b_{1} .\end{cases}
\end{aligned}
$$
hold:

Again, let $\rho_{0}=(1 / 3) \rho_{3}$. Then, the following premises

(1) $\rho_{0}$ is also a metric on $R^{3}, \rho_{0} \leq \rho_{1} \leq \rho_{2} \leq \rho_{3}$, and $\rho_{3}(\vec{a}, \vec{b}) \leq \rho_{4}(\vec{a}, \vec{b})$ if $\vec{a}=\vec{b}$ or $a_{1} \neq b_{1}$ but $\mid\left(a_{2}\right.$ $\left.\left.-b_{2}\right)\left(a_{3}-b_{3}\right) \mid-a_{2} b_{2}-a_{3} b_{3} \leq \sqrt{\left(a_{2}^{2}\right.}+a_{3}^{2}\right)\left(b_{2}^{2}+b_{3}^{2}\right)$ (particularly, $a_{2}=b_{2}$ ).

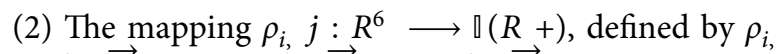
$j(\vec{a}, \vec{b})=\left\langle\rho_{i}(\vec{a}, \vec{b}), \rho_{j}(\vec{a}, \vec{b})\right\rangle$, are intervalvalued metrics on $R^{3}$ for $0 \leq i \leq j \leq 3$, interval-valued metrics on $[0,+\infty)^{3}$ for $0 \leq i \leq j \leq 4$, and intervalvalued metrics on $(-\infty, 0]^{3}$ for $0 \leq i \leq j \leq 4$.

(3) The mapping $\rho_{\mathrm{e}}: R^{6} \longrightarrow \square(R+)$, defined by $\rho_{\mathbf{e}}(\vec{a}$, $\vec{b})=e_{0} \rho_{0}(\vec{a}, \vec{b})+e_{1} \rho_{1}(\vec{a}, \vec{b})+e_{2} \rho_{2}(\vec{a}, \vec{b})$ $+e_{3} \rho_{3}(\vec{a}, \vec{b})+e_{4} \rho_{4}(\vec{a}, \vec{b})\left(e_{i} \geq 0, i=0,1,2,3,4\right)$ is an interval-valued metric (actually, a metric) (Each of these interval-valued metrics can be used to measure the difference between any two bodies $A$ and $B$ with the centroid set $\{\vec{a}, \vec{b}\} \subseteq R^{3}$.) on $R^{3}$.

(4) Let $\left(w_{1}, w_{2}\right) \in[0,1]^{2}$ satisfying $w_{1}+w_{2}=1$. Then, the mappings $\rho, \rho . w, \rho_{w} ., \quad: C([a, b]) \times C([a, b])$ $\longrightarrow \square(R+)$, defined by $\rho(h, g)=\langle h g, \overline{h g}\rangle, \rho . w(h, g)$ $=\left\langle h g, w_{1} h g+w_{2} \overline{h g}\right\rangle$, and $\rho_{w} .(h, \bar{g})=\left\langle w_{1} \underline{h g}+w_{2} \overline{h g}\right.$, $\overline{h g}\rangle(\forall\{h, \bar{g}\} \subseteq C([a, b]))$, are interval-valued metric on $C([a, b])$ (the set of all continuous functions on $[a, b], a$ $<b)$ which can be used to measure the difference between two functions $h, g \in C([a, b])$, where

$$
\begin{aligned}
& \underline{h g}=\frac{1}{b-a} \int_{a}^{b}|f(t)-g(t)| \mathrm{d} t, \\
& \overline{h g}=\max _{t \in[a, b]}|f(t)-g(t)| .
\end{aligned}
$$

Proposition 2. Let $Y \neq \varnothing$ and $\vec{w}=\left(w_{1}, w_{2}\right)$ a weight vector (i.e., it satisfies $\left(w_{1}, w_{2}\right) \in[0,1]^{2}$ and $w_{1}+w_{2}=1$ ). Then, 
(1) Consider three mappings $\bar{d}_{1} w, \bar{d}_{w}, 2, \bar{d}_{12}$ from $\mathscr{Y}_{1}^{2}$ $=[0,1]^{Y} \times[0,1]^{Y}$ to $\square(R+)$, defined by $\left((\Phi, \Psi) \in \mathscr{Y}_{1}^{2}\right) d$ ${ }_{1}, w(\Phi, \Psi)=\left\langle d_{1}(\Phi, \Psi), d_{w}(\Phi, \Psi)\right\rangle$,

$\bar{d}_{w,} 2(\Phi, \Psi)=\left\langle d_{w}(\Phi, \Psi), d_{2}(\Phi, \Psi)\right\rangle$,

$\bar{d}_{1,2}(\Phi, \Psi)=\left\langle d_{1}(\Phi, \Psi), d_{2}(\Phi, \Psi)\right\rangle$, where

$d_{1}(\Phi, \Psi)=\inf \mid \Phi(y)-\Psi(y) \| y \in Y$,

$d_{2}(\Phi, \Psi)=\sup \mid \Phi(y)-\Psi(y) \| y \in Y$,

and $d_{w}(\Phi, \Psi)=w_{1} d_{1}(\Phi, \Psi)+w_{2} d_{2}(\Phi, \Psi)$. The last two are WIVP-metrics on $\mathscr{Y}_{1}$ (but the first is not in general); all of them can be used to measure the difference between any two fuzzy sets $\Phi, \Psi \in \mathcal{Y}_{1}$.

(2) For each $d \in\left\{\rho_{0}, \rho_{1}, \rho_{2}, \rho_{3}, \rho_{4}, \rho_{e}\right\}$, consider three mappings $\bar{d}_{1}, w, \bar{d}_{w}, 2, \bar{d}_{1,2}$ from $\mathscr{Y}_{2}^{2}=\left([0,1]^{2}\right)^{Y}$ $\times\left([0,1]^{2}\right)^{Y}$ to $\square(R+)$, defined by $\left((\Phi, \Psi) \in \mathcal{Y}_{2}^{2}\right) \bar{d}_{1}$, $w(\Phi, \Psi)=\left\langle d_{1}(\Phi, \Psi), d_{w}(\Phi, \Psi)\right\rangle$,

$\bar{d}_{w,} 2(\Phi, \Psi)=\left\langle d_{w}(\Phi, \Psi), d_{2}(\Phi, \Psi)\right\rangle$,

$\bar{d}_{1,2}(\Phi, \Psi)=\left\langle d_{1}(\Phi, \Psi), d_{2}(\Phi, \Psi)\right\rangle$, where

$d_{1}(\Phi, \Psi)=\wedge\{d(\Phi(y), \Psi(y)) \mid y \in Y\}$,

$d_{2}(\Phi, \Psi)=\vee\{d(\Phi(y), \Psi(y)) \mid y \in Y\}$,

$d_{w}(\Phi, \Psi)=w_{1} d_{1}(\Phi, \Psi)+w_{2} d_{2}(\Phi, \Psi)$, and the infimum and supremum are taken in $(\square(R), \leqslant)$. The last two are WIVP-metrics on $\mathscr{Y}_{2}$ (but the first is not in general); all of them can be used to measure the difference between any two interval-valued fuzzy sets, intuitionistic fuzzy sets, or bipolar fuzzy sets [34] $Ф, \Psi$ $\in \mathscr{Y}_{2}$.

(3) Analogously, for each $d \in\left\{\rho_{0}, \rho_{1}, \rho_{2}, \rho_{3}, \rho_{4}, \rho_{\mathrm{e}}\right\}$, consider three mappings $\bar{d}_{1}, w, \bar{d}_{w}, 2, \bar{d}_{1,2}$ from $\mathscr{Y}_{3}$ $=\left([0,1]^{3}\right)^{Y} \times\left([0,1]^{3}\right)^{Y}$ to $\llbracket(R+)$, defined by $((\Phi, \Psi)$ $\left.\in \mathscr{Y}_{3}^{2}\right) \bar{d}_{1} w(\Phi, \Psi)=\left\langle d_{1}(\Phi, \Psi), d_{w}(\Phi, \Psi)\right\rangle$,

$\bar{d}_{w}, 2(\Phi, \Psi)=\left\langle d_{w}(\Phi, \Psi), d_{2}(\Phi, \Psi)\right\rangle$,

$\bar{d}_{1,2}(\Phi, \Psi)=\left\langle d_{1}(\Phi, \Psi), d_{2}(\Phi, \Psi)\right\rangle$, where

$d_{1}(\Phi, \Psi)=\wedge\{d(\Phi(y), \Psi(y)) \mid y \in Y\}$,

$d_{2}(\Phi, \Psi)=\vee\{d(\Phi(y), \Psi(y)) \mid y \in Y\}$,

$d_{w}(\Phi, \Psi)=w_{1} d_{1}(\Phi, \Psi)+w_{2} d_{2}(\Phi, \Psi)$, and the infimum and supremum are taken in $(\square(R), \leqslant)$ (Of course, the infimal and supremal in (2) and (3) can also be taken in $(\square(R), \leq))$. The last two are WIVP-metrics on $\mathscr{Y}_{3}$ (but the first is not in general); all of them can be used to measure the difference between any two q-rung orthopair fuzzy sets $(q \geq 1)$ [35-40] or 3-polar fuzzy sets [34] $\Phi, \Psi \in \mathcal{Y}_{3}$.

Proof. We only show that $d_{w}$ in (1) does not satisfy the triangle inequality in general. Actually, take $U=\{x, y\}, A$

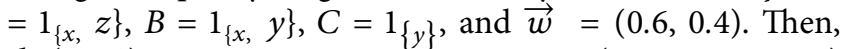
$d_{w}(A, C)=0.6 \times 1+0.4 \times 1 \stackrel{1}{=} 1 \leq 0.8=(0.6 \times 0+0.4 \times 1)$ $+(0.6 \times 0+0.4 \times 1)=d_{w}(A, B)+d_{w}(B, C)$.

\section{Example 2}

(1) Let (The data needed here can also be taken from Example 5.) $A=\{(0.1 / x),(0.2 / y),(0.1 / z)\}$ and $B=\{(1$ $(x),(0.9 / y),(1 / z)\}$ be feature fuzzy sets representing two physical entities (e.g., patients), and $C=\{(0.6 / x)$, $(0.5 / y),(0.6 / z)\}$ the third.
If we take a weight vector $\vec{w}=(0.5,0.5)$, as $\bar{d}_{1,2}(C, A)$ $=\langle 0.3,0.5\rangle \leq 0.4=\bar{d}_{1,2}(C, B), C$ belongs to class $A$ (with respect to $\bar{d}_{1,2}$ ); as $\bar{d}_{1} w(C, A)=\langle 0.3,0.4\rangle \leq 0.4$ $=\bar{d}_{1,2}(C, B), C$ belongs to class $A$ (with respect to $\bar{d}_{1,2}$ ); as $\bar{d}_{w}, 2(C, B)=0.4 \leq\langle 0.4,0.5\rangle=\bar{d}_{w}, 2(C, A), C$ belongs to class $B$ (with respect to $\bar{d}_{w}, 2$ ).

If we take two weight vectors $\vec{w}=(0.5,0.5)$ and $v$ $=(0.4,0.6)$, then $d_{w}(C, A)=0.5 \times 0.3+0.5 \times 0.5=0.4$, $d_{v}(C, A)=0.4 \times 0.3+0.6 \times 0.5=0.42, d_{w}(C, B)=0.5$ $\times 0.4+0.5 \times 0.4=0.4$, and $d_{v}(C, B)=0.4 \times 0.4+0.6 \times 0.4$ $=0.4$. Thus, $d_{w v}(C, A)=\langle 0.4,0.42\rangle$ and $d_{w v}(C, B)$ $=0.4$. As $\bar{d}_{1} w v(C, A)=\langle 0.3,\langle 0.4,0.42\rangle\rangle \leq 0.4=\bar{d}_{1}$, $w v(C, B), C$ belongs to class $A$ (with respect to $\bar{d}_{1}, w v$ ); as $\bar{d}_{w} 2(C, B)=0.4 \leq\langle\langle 0.4,0.42\rangle, 0.5\rangle=\bar{d}_{w} 2(C, A)$, $C$ belongs to class $B$ (with respect to $\bar{d}_{w}, 2$ ), where $\leq$ is the point-wise order on $\square(R+) \times \square(R+)$ induced by $\leq$ on $\mathbb{\square}(R+)$.

(2) Let $A=\{(\langle 0.1,0.2\rangle / x),(\langle 0.3,0.4\rangle / y),(\langle 0.1,0.2\rangle$

$\mid z)\}$ and $B=\{(\langle 0.9,1\rangle / x),(\langle 0.8,0.9\rangle / y),(\langle 0.9,1\rangle$

$\mid z)\}$ be feature interval-valued fuzzy sets representing two physical entities (e.g., patients), $C=\{(0.6 / x)$, $(\langle 0.4,0.6\rangle / y),(0.6 / z)\}$ the third, and $\vec{w}=(0.5,0.5)$. As $\left(\overline{\rho_{0}}\right)_{1,2}(C, A)=\langle 0.1,0.3\rangle \leq(7 / 30)=\left(\overline{\rho_{0}}\right)_{1,2}(C, B)$, $C$ belongs to class $A$ (with respect to $\left.\left(\overline{\rho_{0}}\right)_{1,2}\right)$; as $\left(\overline{\rho_{0}}\right)_{1,2}(C, A)=\langle 0.1,0.2\rangle \leq(7 / 30)=\left(\overline{\rho_{0}}\right)_{1}, w(C, B), C$ belongs to class $A$ (with respect to $\left(\overline{\rho_{0}}\right)_{1}, w$ ); as $\left(\overline{\rho_{0}}\right)_{w}$, $2(C, B)=(7 / 30) \leq\langle 0.2,0.3\rangle=\left(\overline{\rho_{0}}\right)_{w}, 2(C, A), C$ belongs to class $B$ (with respect to $\left(\bar{\rho}_{0}\right)_{w}, 2$ ).

As $\left(\overline{\rho_{1}}\right)_{1,2}(C, A)=\langle 0.2,0.5\rangle \leq 0.4=\left(\overline{\rho_{1}}\right)_{1,2}(C, B), C$ belongs to class $A$ (with respect to $\left.\left(\overline{\rho_{1}}\right)_{1,2}\right)$; as $\left(\overline{\rho_{1}}\right)_{1}$, $w(C, A)=\langle 0.2,0.35\rangle \leq 0.4=\left(\overline{\rho_{1}}\right)_{1}, w(C, B), C$ belongs to class $A$ (with respect to $\left.\left(\overline{\rho_{1}}\right)_{1}, w\right)$; as $\left(\overline{\rho_{1}}\right)_{w}, 2(C, B)$ $=0.4 \leq\langle 0.35,0.5\rangle=\left(\overline{\rho_{1}}\right)_{w}, 2(C, A), C$ belongs to class $B$ (with respect to $\left(\overline{\rho_{1}}\right)_{w}, 2$ ).

As $\left(\overline{\rho_{2}}\right)_{1,2}(C, A)=\langle\sqrt{0.05}, \sqrt{0.41}\rangle \leq 0.5=\left(\overline{\rho_{2}}\right)_{1,2}(C$, $B$ ), $C$ belongs to class $A$ (with respect to $\left.\left(\overline{\rho_{2}}\right)_{1,2}\right)$; as $(\bar{\rho}$ $\left.{ }_{2}\right)_{1}, w(C, A)=\langle\sqrt{0.05}, 0.5(\sqrt{0.05}+\sqrt{0.41})\rangle \leq 0.5=$ $\left(\overline{\rho_{2}}\right)_{1}, w(C, B), C$ belongs to class $A$ (with respect to $\left(\overline{\rho_{2}}\right.$ )$\left._{1}, w\right)$; as $\left(\overline{\rho_{2}}\right)_{w}, 2(C, B)=0.5 \leq\langle 0.5(\sqrt{0.05}+\sqrt{0.41})$, $\sqrt{0.41}\rangle=\left(\overline{\rho_{2}}\right)_{w}, 2(C, A), C$ belongs to class $B$ (with respect to $\left.\left(\overline{\rho_{2}}\right)_{w}, 2\right)$.

As $\left(\overline{\rho_{3}}\right)_{1,2}(C, A)=\langle 0.3,0.9\rangle \leq 0.7=\left(\overline{\rho_{3}}\right)_{1,2}(C, B), C$ belongs to class $A$ (with respect to $\left.\left(\overline{\rho_{3}}\right)_{1,2}\right)$; as $\left(\overline{\rho_{3}}\right)_{1}$, $w(C, A)=\langle 0.3,0.6\rangle \leq 0.7=\left(\overline{\rho_{3}}\right)_{1}, w(C, B), C$ belongs to class $A$ (with respect to $\left.\left(\overline{\rho_{3}}\right)_{1}, w\right)$; as $\left(\overline{\rho_{3}}\right)_{w}, 2(C, B)$ $=0.7 \leq\langle 0.6,0.9\rangle=\left(\overline{\rho_{3}}\right)_{w}, 2(C, A), C$ belongs to class $B$ (with respect to $\left(\overline{\rho_{3}}\right)_{w}, 2$ ).

As $\left(\overline{\rho_{4}}\right)_{1,2}(C, A)=\langle 1.1,1.3\rangle \leq 1.9=\left(\overline{\rho_{4}}\right)_{1,2}(C, B), C$ belongs to class $A$ (with respect to $\left.\left(\overline{\rho_{4}}\right)_{1,2}\right) ;$ as $\left(\overline{\rho_{4}}\right)_{1}$, $w(C, A)=\langle 1.1,1.2\rangle \leq 1.9=\left(\overline{\rho_{4}}\right)_{1}, w(C, B), C$ belongs to class $A$ (with respect to $\left.\left(\overline{\rho_{4}}\right)_{1}, w\right)$; as $\left(\overline{\rho_{4}}\right)_{w}, 2(C, A)$ $=\langle 1.2,1.3\rangle \leq 1.9=\left(\overline{\rho_{4}}\right)_{w}, 2(C, B), C$ belongs to class $A$ (with respect to $\left(\overline{\rho_{4}}\right)_{w}, 2$ ).

It follows that $\left(\bar{\rho}_{\mathbf{e}}\right)_{1,2}(C, A) \leq\left(\bar{\rho}_{\mathbf{e}}\right)_{1,2}(C, B)$ and $\left(\bar{\rho}_{\mathbf{e}}\right)_{1}$, $w(C, A) \leq\left(\bar{\rho}_{\mathrm{e}}\right)_{1}, w(C, B)$ for each $\left(e_{0}, e_{1}, e_{2}, e_{3}, e_{4}\right)$ $\in R^{5}$, thus $C$ belongs to class $A$ (with respect to $\left(\bar{\rho}_{\mathbf{e}}\right)_{1,2}$ 
and $\left.\left(\bar{\rho}_{\mathrm{e}}\right)_{1} w\right)$. Take $e_{0}=e_{1}=e_{2}=e_{3}=e_{4}=0.2$. Then, $\left(\bar{\rho}_{\mathbf{e}}\right)_{w} 2(C, A) \leq\left(\bar{\rho}_{\mathbf{e}}\right)_{w}, 2(C, B)$, thus $C$ belongs to class $A$ (with respect to $\left.\left(\bar{\rho}_{\mathbf{e}}\right)_{w}, 2\right)$.

(3)Let $A=\{(\langle 0.1,0.2,0.1\rangle / x), \quad(\langle 0.3,0.4,0.3\rangle / y)$, $(\langle 0.1,0.2,0.1\rangle / z)\}$ and $B=\{(\langle 0.8,0.1,0.1\rangle / x),(\langle 0.7$, $0.2,0.1\rangle / y),(\langle 0.8,0.1,0.1\rangle / z)\}$ be feature picture fuzzy sets representing two physical entities (e.g., patients), $C$ $=\{((0.6,0.1,0.1) / x),(\langle 0.5,0.1,0\rangle / y),((0.6,0.1,0.1)$ $\mid z)\}$ the third, and $\vec{w}=(0.5,0.5)$.

As $\left(\overline{\rho_{0}}\right)_{1,2}(C, A)=(1 / 3)\langle 0.2,0.4\rangle \leq(1 / 3)\langle 0.6,0.8\rangle$ $=\left(\bar{\rho}_{0}\right)_{1,2}(C, B), C$ belongs to class $A$ (with respect to $\left.\left(\overline{\rho_{0}}\right)_{1,2}\right) ;$ as $\left(\overline{\rho_{0}}\right)_{1}, w(C, A)=(1 / 3)\langle 0.2,0.3\rangle \leq(1 /$ $3)\langle 0.6,0.7\rangle=\left(\overline{\rho_{0}}\right)_{1}, w(C, B), C$ belongs to class $A$ (with respect to $\left.\left(\overline{\rho_{0}}\right)_{1}, w\right)$; as $\left(\overline{\rho_{0}}\right)_{w}, 2(C, A)=(1 / 3)\langle 0.3,0.4\rangle$ $\leq(1 / 3)\langle 0.7,0.8\rangle=\left(\overline{\rho_{0}}\right)_{w}, 2(C, B), C$ belongs to class $A$ (with respect to $\left(\overline{\rho_{0}}\right)_{w}$ ).

As $\left(\overline{\rho_{1}}\right)_{1,2}(C, A)=0.2 \leq\langle 0.3,0.5\rangle=\left(\overline{\rho_{1}}\right)_{1,2}(C, B), C$ belongs to class $A$ (with respect to $\left.\left(\overline{\rho_{1}}\right)_{1,2}\right)$; as $\left(\overline{\rho_{1}}\right)_{1}$, $w(C, A)=0.2 \leq\langle 0.3,0.4\rangle=\left(\overline{\rho_{1}}\right)_{1}, w(C, B), C$ belongs to class $A$ (with respect to $\left.\left(\overline{\rho_{1}}\right)_{1}, w\right)$; as $\left(\overline{\rho_{1}}\right)_{w}, 2(C, A)$ $=0.2 \leq\langle 0.4,0.5\rangle=\left(\overline{\rho_{1}}\right)_{w}, 2(C, B), C$ belongs to class $A$ (with respect to $\left(\overline{\rho_{1}}\right)_{w}, 2$ ).

As $\left(\overline{\rho_{2}}\right)_{1,2}(C, A)=\langle 0.2, \sqrt{0.06}\rangle \leq\langle\sqrt{0.22}, \sqrt{0.26}\rangle$ $=\left(\rho_{2}\right)_{1,2}(C, B), C$ belongs to class $A$ (with respect to $\left.\left(\overline{\rho_{2}}\right)_{1,2}\right)$; as $\left(\overline{\rho_{2}}\right)_{1}, w(C, A)=\langle 0.2,0.5(0.2+\sqrt{0.06})\rangle$ $\leq\langle\sqrt{0.22}, 0.5(\sqrt{0.22}+\sqrt{0.26})\rangle=\left(\overline{\rho_{2}}\right)_{1}, w(C, B), C$ belongs to class $A$ (with respect to $\left.\left(\rho_{2}\right)_{1}, w\right)$; as $\left(\bar{\rho}_{2}\right)_{w}$, $2(C, A)=\langle 0.5(0.2+\sqrt{0.06}), \sqrt{0.06}\rangle \leq\langle 0.5(\sqrt{0.22}$ $+\sqrt{0.26}), \sqrt{0.26}\rangle=\left(\overline{\rho_{2}}\right)_{w}, 2(C, B), C$ belongs to class $A$ (with respect to $\left(\overline{\rho_{2}}\right)_{w}, 2$ ).

As $\left(\overline{\rho_{3}}\right)_{1,2}(C, A)=\langle 0.2,0.4\rangle \leq\langle 0.6,0.8\rangle=\left(\overline{\rho_{3}}\right)_{1,2}(C$, $B$ ), $C$ belongs to class $A$ (with respect to $\left.\left(\overline{\rho_{3}}\right)_{1,2}\right)$; as $\left(\overline{\rho_{3}}\right)_{1}, w(C, A)=\langle 0.2,0.3\rangle \leq\langle 0.6,0.7\rangle=\left(\overline{\rho_{3}}\right)_{1}, w(C$, $B$ ), $C$ belongs to class $A$ (with respect to $\left(\overline{\rho_{3}}\right)_{1}, w$ ); as $\left(\overline{\rho_{3}}\right)_{w}, 2(C, A)=\langle 0.3,0.4\rangle \leq\langle 0.7,0.8\rangle=\left(\overline{\rho_{3}}\right)_{w}, 2(C$, $B$ ), $C$ belongs to class $A$ (with respect to $\left(\overline{\rho_{3}}\right)_{w}, 2$ ).

As $\left(\overline{\rho_{4}}\right)_{1,2}(C, A)=\langle 0.2+2 \sqrt{0.02}, 0.2+\sqrt{0.05}$ $+\sqrt{0.01}\rangle \leq\langle 0.5+2 \sqrt{0.02}, 0.9+\sqrt{0.05}\rangle=\left(\overline{\rho_{4}}\right)_{1,2}(C$,
$B$ ), $C$ belongs to class $A$ (with respect to $\left.\left(\overline{\rho_{4}}\right)_{1,2}\right)$; as $\left(\overline{\rho_{4}}\right)_{1}, w(C, \quad A)=\langle 0.2+2 \sqrt{0.02}, \quad 0.2+\sqrt{0.02}$ $+0.5(\sqrt{0.05}+\sqrt{0.01})\rangle \leq\langle 0.5+2 \sqrt{0.02}, 0.45+\sqrt{0.02}$ $+0.5 \sqrt{0.05}\rangle=\left(\overline{\rho_{4}}\right)_{1}, w(C, B), C$ belongs to class $A$ (with respect to $\left.\left(\bar{\rho}_{4}\right)_{1}, w\right)$; as $\left(\bar{\rho}_{4}\right)_{w}, 2(C, A)=\langle 0.2$ $+\sqrt{0.02}+0.5(\sqrt{0.05}+\sqrt{0.01}), 0.2+\sqrt{0.05}+\sqrt{0.01}\rangle$ $\leq\langle 0.45+\sqrt{0.02}+0.5 \sqrt{0.05}, 0.9+\sqrt{0.05}\rangle=\left(\overline{\rho_{4}}\right)_{w}$, $2(C, B), C$ belongs to class $A$ (with respect to $\left.\left(\overline{\rho_{4}}\right)_{w}, 2\right)$. It follows $\left(\bar{\rho}_{\mathbf{e}}\right)_{1,2}(C, A) \leq\left(\bar{\rho}_{\mathbf{e}}\right)_{1,2}(C, B),\left(\bar{\rho}_{\mathbf{e}}\right)_{1}, w(C, A)$ $\leq\left(\bar{\rho}_{\mathbf{e}}\right)_{1}, w(C, B)$, and $\left(\bar{\rho}_{\mathbf{e}}\right)_{w}, 2(C, A) \leq\left(\bar{\rho}_{\mathbf{e}}\right)_{w}, 2(C, B)$ for each $\left(e_{0}, e_{1}, e_{2}, e_{3}, e_{4}\right) \in R^{5}$, thus $C$ belongs to class $A$ (with respect to $\left(\bar{\rho}_{\mathbf{e}}\right)_{1,2},\left(\bar{\rho}_{\mathbf{e}}\right)_{1}, w$, and $\left.\left(\bar{\rho}_{\mathbf{e}}\right)_{w}, 2\right)$.

(4)Let $A=\{(\langle 0.1,0.2,0.1\rangle / x),(\langle 0.3,0.4,0.3\rangle / y)$, $(\langle 0.1,0.2,0.1\rangle / z)\}$ and $B=\{(\langle 0.9,0.1,0.1\rangle / x),(\langle 0.8$, $0.2,0.1\rangle / y),(\langle 0.9,0.1,0.1\rangle / z)\}$ be feature generalized pythagorean fuzzy sets (A generalized pythagorean fuzzy set is a special 3-polar fuzzy set $A: X \longrightarrow[0,1]^{3}$ satisfying $\rho_{2}(A(x), \overrightarrow{0}) \leq 1(\forall x \in X)$.) (see [38, 39] for a special case) representing two physical entities (e.g., patients $), C=\{(0.6,0.1,0.1 / x),(\langle 0.5,0.1,0\rangle / y)$, $(\langle 0.6,0.1,0.1\rangle / z)\}$ the third, and $\vec{w}=(0.5,0.5)$. Similar to (3), as $\left(\overline{\rho_{0}}\right)_{1,2}(C, B)=(1 / 3)\langle 0.3,0.5\rangle \leq(1$ $13)\langle 0.2,0.8\rangle=\left(\overline{\rho_{0}}\right)_{1,2}(C, A), C$ belongs to class $B$ (with respect to $\left.\left(\overline{\rho_{0}}\right)_{1,2}\right)$. Analogously, we can decide $C$ belongs to class $A$ or $B$ with respect to $\left(\overline{\rho_{0}}\right)_{1}, w$ (resp., $\left(\overline{\rho_{0}}\right)_{w}, 2,\left(\overline{\rho_{1}}\right)_{1,2},\left(\overline{\rho_{1}}\right)_{1}, w,\left(\overline{\rho_{1}}\right)_{w}, 2,\left(\overline{\rho_{2}}\right)_{1,2},\left(\overline{\rho_{2}}\right)_{1}, w$, $\left(\overline{\rho_{2}}\right)_{w}, 2,\left(\overline{\rho_{3}}\right)_{1,2},\left(\overline{\rho_{3}}\right)_{1}, w,\left(\overline{\rho_{3}}\right)_{w}, 2,\left(\overline{\rho_{4}}\right)_{1,2},\left(\overline{\rho_{4}}\right)_{1}, w$, $\left.\left(\overline{\rho_{4}}\right)_{w}, 2\right)$.

\section{Example 3}

(1) Analogously, we can predict $A(n+1)$ from a given time series $A(1), A(2), \ldots, A(n)(n>3)$ in the following way: Firstly, fix (maybe by experts) a natural number $m<n$ (say, $m=3$ ) and a set $\left\{w_{1}, w_{2}\right.$, $\left.w_{3}\right\} \subseteq(0,1)$ satisfying $w_{1}<w_{2}<w_{3}$ and $w_{1}+w_{2}+w_{3}$ $=1$. Secondly, compute the differences $r_{i}(i=1,2$, $\ldots, n-3)$ :

$$
\begin{aligned}
r_{i} & =\rho((A(i), A(i+1), A(i+2)),(A(n-2), A(n-1), A(n))) \\
& =w_{1}|A(n-2)-A(i)|+w_{2}|A(n-1)-A(i+1)|+w_{3}|A(n)-A(i+2)| .
\end{aligned}
$$

Finally, take $A(n+1)=A(k+3)$ if $r_{k} \leq r_{i}(i=1,2, \ldots$, $n-3)$. We can also compute each $r_{i}$ as Example 2.

(2)(Continued to Proposition $1(4))$ Let $f(t)=t$ and $g(t)=t^{3}$ be feature functions representing two physical entities, and $h(t)=t^{2}(t \in[0,1])$ the third. Then, $h f$ $=\int_{0}^{1}\left(t-t^{2}\right) \mathrm{d} t=(1 / 2)-(1 / 3)=(1 / 6), \underline{h g}=\int_{0}^{1}\left(t^{2}\right.$ $\left.-t^{3}\right) \mathrm{d} t=(1 / 3)-(1 / 4)=(1 / 12), \overline{h f}=(1 / 4)$, and $\overline{h g}$ $=(4 / 27)$.

If we take a weight vector $\vec{w}=(0.5,0.5)$, as $\rho(h, g)=\langle(1$ /12), $(4 / 27)\rangle \leq\langle(1 / 6),(1 / 4)\rangle=\rho(h, f), h$ belongs to class $g$ (with respect to $\rho)$; as $\rho . w(h, g)=\langle(1 / 12),(25 / 216)\rangle$ $\leq\langle(1 / 6),(5 / 24)\rangle=\rho . w(h, f), h$ belongs to class $g$ (with respect to $\rho)$; as $\rho_{w} \cdot(h, g)=\langle(25 / 216),(4 / 27)\rangle \leq\langle(5 / 24)$, $(1 / 4)\rangle=\rho_{w} .(h, f), h$ belongs to class $g$ (with respect to $\rho$ ).

If we take two weight vectors $\vec{w}=(0.5,0.5)$ and $\vec{v}$ $=(0.6,0.4)$, then $\rho . v(h, f)=\langle(1 / 6),(1 / 5)\rangle, \rho . v(h, g)$ $=\langle(1 / 12),(59 / 540)\rangle, \rho_{v} .(h, f)=\langle(1 / 5),(1 / 4)\rangle$, and $\rho_{\nu .}(h$, $g)=\langle(59 / 540),(4 / 27)\rangle$. Therefore, $\rho . v w(h, f)=0.5\langle(1$ /6), $(1 / 5)\rangle \oplus 0.5\langle(1 / 6),(5 / 24)\rangle=\langle(1 / 6),(49 / 240)\rangle, \rho$. $v w(h, g)=0.5\langle(1 / 12),(59 / 540)\rangle \oplus 0.5\langle(1 / 12),(25 / 216)\rangle$ $=\langle(1 / 12),(89 / 1296)\rangle, \rho_{v w} .(h, f)=0.5\langle(1 / 5),(1 / 4)\rangle$ $\oplus 0.5\langle(5 / 24), \quad(1 / 4)\rangle=\langle(49 / 240),(1 / 4)\rangle, \rho_{v w} .(h, g)=$ 
$0.5\langle(59 / 540), \quad(7 / 24)\rangle \oplus 0.5\langle(25 / 216), \quad(4 / 27)\rangle=\langle(89$ /1296), (11/54)〉. As $\rho . v w(h, g) \leq \rho . v w(h, f), h$ belongs to class $g$ (with respect to $\rho . v w$ ); as $\rho_{v w}$. $(h, g) \leq \rho_{v w}$. $(h, f), h$ belongs to class $g$ (with respect to $\rho . v w$ ).

\section{Proposition 3}

(1) Let $\mathscr{A} \subseteq 2^{X}-\{\varnothing\}(X \neq \varnothing, \mathscr{A} \neq \varnothing)$. If $\rho-($ resp., $\rho+)$ is a pseudo-semi-metric (resp., a pseudo-metric) on $\mathscr{A}$ satisfying $\rho-(A, B) \leq \rho+(A, B)(\{A, B\} \subseteq \mathscr{A})$, then $\tilde{\rho}$ $: \mathscr{A}^{2} \longrightarrow \square(R+)$, defined by $\widetilde{\rho}(A, B)=\langle\rho-(A, B), \rho$ $+(A, B)\rangle(\{A, B\} \subseteq \mathscr{A})$, is a WIVP-metric on $\mathscr{A}$.

(2) If $\varrho_{1}$ and $\varrho_{2}$ are pseudo-metrics on $X(X \neq \varnothing)$, then $\varrho_{1} \wedge \varrho_{2}$, defined by $\left(\varrho_{1} \wedge \varrho_{2}\right)(x, y)=\min \left\{\varrho_{1}(x, y), \varrho_{2}(x\right.$, $y)\}$, is a pseudo-semi-metric on $X, \varrho_{1} \vee \varrho_{2}$, defined by $\left(\varrho_{1}\right.$ $\left.\vee \varrho_{2}\right)(x, y)=\max \left\{\varrho_{1}(x, y), \varrho_{2}(x, y)\right\}$, is a pseudometric on $X$, and thus $\varrho_{1} \Delta \varrho_{2}$, defined by

$$
\left(\varrho_{1} \Delta \varrho_{2}\right)(x, y)=\left\langle\left(\varrho_{1} \wedge \varrho_{2}\right)(x, y),\left(\varrho_{1} \vee \varrho_{2}\right)(x, y)\right\rangle,
$$

is a WIVP-metric on $X$.

(3) Let $\mathscr{A}=\left\{A \in 2^{R^{3}}-\{\varnothing\} \mid A\right\}$ is connected and bounded with respect to the ordinary Euclidean metric on $R^{3}$, and Lebesgue measurable. Then, $d_{0}$, defined by $d_{0}(A, B)=\inf \left\{\rho_{2}(\vec{x}, \vec{y}) \mid \vec{x} \in A, \vec{y} \in B\right\}\left(\rho_{2}\right.$ is the ordinary Euclidean metric on $R^{3}$ ), is a pseudo-semimetric; $d_{1}$, defined by $d_{1}(A, B)=\rho_{2}\left(c_{A}, c_{B}\right)$, is a pseudometric; and $d_{2}$, defined by

$$
d_{2}(A, B)=\max \left\{\sup _{\vec{a} \in A} \inf _{\vec{b} \in B} \rho_{2}(\vec{a}, \vec{b}), \sup _{\vec{b} \in B} \inf _{\vec{a} \in A} \rho_{2}(\vec{a}, \vec{b})\right\}
$$

is a metric (called the Hausdorff metric induced by $\rho_{2}$ and written also as $\left.\rho_{2}^{H}\right)$. Therefore, we obtain three WIVP-metrics $d_{i}: \mathscr{A}^{2} \longrightarrow \square(R+)(i=1,2,3)$ on $\mathscr{A}$ each of which can be used to measure the difference between any two heavenly bodies $A$ and $B$ in $\mathscr{A}$ with the centroids $\left\{c_{A}, c_{B}\right\} \subseteq R^{3}$, where $d_{i}(A, B)=\left\langle d_{0}(A, B)\right.$, $\left.d_{i}(A, B)\right\rangle(i=1,2)$ and $d_{3}(A, B)=\left(d_{1} \Delta d_{2}\right)(A, B)=$ $\left\langle\min \left\{d_{1}(A, B), d_{2}(A, B)\right\}, \max \left\{d_{1}(A, B), d_{2}(A, B)\right\}\right\rangle$. As $d_{0}(A, B) \leq \min \left\{d_{1}(A, B), d_{2}(A, B)\right\}, d_{1}(A, B)$ $V d_{2}(A, B) \leq d_{3}(A, B)\left(\forall(A, B) \in \mathscr{A}^{2}\right)$.

(4)Let $\rho$ be a pseudo-metric on $\mathscr{A} \subseteq 2^{X}-\{\varnothing\}(X \neq \varnothing, \mathscr{A}$ $\neq \varnothing), \mathscr{A}=\left\{\Phi \in L^{X} \mid \Phi_{[d]} \in \mathscr{A}(\forall d \in L)\right\}$ (where (L, $\leq)$ is a poset), and $\{a, c\} \subseteq L$. Then, the mapping $\rho_{a c}: \mathscr{A}^{2}$ $\longrightarrow \square(R+)$, defined by $(\{\Phi, \Psi\} \subseteq \mathscr{A}) \rho_{a c}(\Phi, \Psi)$ $=\left\langle\rho\left(\Phi_{[a]}, \Psi_{[a]}\right) \wedge \rho\left(\Phi_{[c]}, \Psi_{[c]}\right), \rho\left(\Phi_{[a]}, \Psi_{[a]}\right) \vee \rho\left(\Phi_{[c]}\right.\right.$, $\left.\left.\Psi_{[c]}\right)\right\rangle$, is a WIVP-metric on $\mathscr{A}$.

Proof. (1) follows from Definition 3, (2) can be proved by definition, and (3) follows from (2). (4) Defined $\varrho_{1}, \varrho_{2}: \mathscr{A}^{2}$ $\longrightarrow R+$ by putting $\varrho_{1}(\Phi, \Psi)=\rho\left(\Phi_{[a]}, \Psi_{[a]}\right)$ and $\varrho_{2}(\Phi, \Psi)$ $=\rho\left(\Phi_{[c]}, \Psi_{[c]}\right)(\{\Phi, \Psi\} \subseteq \mathscr{A})$. Then, $\rho_{a c}=\varrho_{1} \Delta \varrho_{2}$ and $\varrho_{1}$ and $\varrho_{2}$ are pseudo-metrics on $\mathscr{A}$, thus $\rho_{a c}$ is a WIVP-metric on $\mathscr{A}$ by (2).

\section{Example 4}

(1) Consider the two bodies $A$ and $B$ in Example 1 (2). Then, the differences between $A$ and $B$ can be taken as (the first three are real numbers and the last three are interval numbers): $d_{0}(A, B)=0, d_{1}(A, B)=\epsilon+\varepsilon, d_{2}(A$, $B)=2 \max \{\epsilon, \varepsilon\}, \mathbf{d}_{1}(A, B)=\langle 0, \epsilon+\varepsilon\rangle, \mathbf{d}_{2}(A, B)=$ $\langle 0,2 \max \{\epsilon, \varepsilon\}\rangle$, or $\mathbf{d}_{3}(A, B)=\langle\epsilon+\mathcal{E}, 2 \max \{\epsilon, \varepsilon\}\rangle$.

(2) Let $\mathscr{A}$ be the set of all nonempty compact sets in the ordinary Euclidean plane $\left(R^{2}, \varrho\right), \rho=\varrho^{H}$ be the Hausdorff metric on $\mathscr{A}$ induced by $\varrho, \mathscr{A}=\{\Phi$
$\left.\in[0,1]^{R^{2}} \mid \Phi_{[d]} \in \mathscr{A}(\forall d \in(0,1])\right\},(a, c)=(0.4,0.6)$, and $(\Phi, \Psi) \in \mathscr{A}$ be two clouds (in which $\Phi(x, y)$ stands for the degree of the thickness of cloud in vertical line through the point $(x, y)$ in the plane $\left.R^{2}\right)$, defined by $\Phi(x, y)=\varphi(x) \wedge \varphi(y)$ (i.e., $\min \{\varphi(x)$, $\varphi(y)\})$ and $\Psi(x, y)=\psi(x) \wedge \psi(y)\left((x, y) \in R^{2}\right)$, where $\varphi(x)=1-|x|(x \in R)$ and $\psi(x)=1-|x-9|(x \in R)$. Then, $\Phi_{[a]}=[-0.6,0.6]^{2}, \Psi_{[a]}=[8.4,9.6]^{2}, \Phi_{[c]}=[$ $-0.4,0.4]^{2}, \Psi_{[c]}=[8.6,9.4]^{2}, \rho\left(\Phi_{[a]}, \Psi_{[a]}\right)=\sqrt{(9.6}$ $-0.6)^{2}+(9.6-0.6)^{2}=9 \sqrt{2}$, and $\rho\left(\Phi_{[c]}, \Psi_{[c]}\right)=\sqrt{(9.4}$ $-0.4)^{2}+(9.4-0.4)^{2}=9 \sqrt{2}$. Therefore, $\rho_{a c}(\Phi, \Psi)$ $=\langle 9 \sqrt{2}, 9 \sqrt{2}\rangle=9 \sqrt{2}$.

(3)Analogously, let $\mathscr{A}$ be the set of all nonempty compact sets in the ordinary Euclidean space $\left(R^{3}, \varrho\right), \rho=\varrho^{H}$ be the Hausdorff metric on $\mathscr{A}$ induced by $\varrho, \mathscr{A}=\{\Phi \in$ $\left.[0,1]^{R^{3}} \mid \Phi_{[d]} \in \mathscr{A}(\forall d \in(0,1])\right\},(a, c)=(0.4,0.6)$, and $(\Phi, \Psi) \in \mathscr{A}$ be two clouds (in which $\Phi(x, y, z)$ stands for the degree of concentration of cloud at the point $(x, y, z))$, defined by $\Phi(x, y, z)=\varphi(x) \wedge \varphi(y) \wedge \varphi(z)$ and $\Psi(x, y, z)=\psi(x) \wedge \psi(y) \wedge \psi(z)\left((x, y, z) \in R^{3}\right)$, where $\varphi(x)=1-|x|(x \in R)$ and $\psi(x)=1-|x-9|(x$ $\in R)$. Then $\Phi_{[a]}=[-0.6,0.6]^{3}, \Psi_{[a]}=[8.4,9.6]^{3}, \Phi_{[c]}$ $=[-0.4,0.4]^{3}, \Psi_{[c]}=[8.6,9.4]^{3}, \rho\left(\Phi_{[a]}, \Psi_{[a]}\right)=\sqrt{3(9.6}$ $-0.6)^{2}=9 \sqrt{3}$, and $\left.\rho\left(\Phi_{[c]}, \Psi_{[c]}\right)=\sqrt{3(9.4}-0.4\right)^{2}=9 \sqrt{3}$. Therefore, $\rho_{a c}(\Phi, \Psi)=\langle 9 \sqrt{3}, 9 \sqrt{3}\rangle=9 \sqrt{3}$.

Example 5. Now, we exemplify an application of the idea of WIVP-metrics in pattern recognition (i.e., COVID-19 diagnosis). Consider the following symptom data (see Table 1), involving 5 symptoms (asthma, sore throat, cough, fever, age), and diagnosis results, involving yes (briefly, $Y$ ) and no (briefly, $N$ ), of 7 patients given by expert physicians in a Chinese hospital in Nanjing:

From this, we can get the following 7 mappings (patients): 
TABLE 1: Symptom data and diagnosis results.

\begin{tabular}{lccccccc}
\hline- & $p_{1}$ & $p_{2}$ & $p_{3}$ & $p_{4}$ & $p_{5}$ & $p_{6}$ & $p_{7}$ \\
\hline Asthma & 39 & 41 & 52 & 45 & 39 & 55 & 60 \\
Sore throat & 48 & 51 & 48 & 53 & 62 & 55 & 57 \\
Cough & 18 & 41 & 32 & 44 & 49 & 36 & 37 \\
Fever & 37.8 & 38 & 38.5 & 37.8 & 40 & 38 & 38.5 \\
Age & 67 & 41 & 44 & 68 & 70 & 50 & 54 \\
Result & $N$ & $Y$ & $N$ & $Y$ & $Y$ & $Y$ & $Y$ \\
\hline
\end{tabular}

$$
\begin{aligned}
& P_{1}=\left\{\frac{39}{\text { asthma }}, \frac{48}{\text { sorethroat }}, \frac{18}{\text { cough }}, \frac{37.8}{\text { fever }}, \frac{67}{\text { age }}\right\}, \\
& P_{2}=\left\{\frac{41}{\text { asthma }}, \frac{51}{\text { sorethroat }}, \frac{41}{\text { cough }}, \frac{38}{\text { fever }}, \frac{41}{\text { age }}\right\}, \\
& P_{7}=\left\{\frac{60}{\text { asthma }}, \frac{57}{\text { sorethroat }}, \frac{37}{\text { cough }}, \frac{38.5}{\text { fever }}, \frac{54}{\text { age }}\right\} .
\end{aligned}
$$

Take other 2 patients in the same hospital for test:

$$
\begin{aligned}
& P_{8}=\left\{\frac{\langle 59,63\rangle}{\text { asthma }}, \frac{60}{\text { sorethroat }}, \frac{28}{\text { cough }}, \frac{37.9}{\text { fever }}, \frac{67}{\text { age }}\right\}, \\
& P_{9}=\left\{\frac{\langle 60,64\rangle}{\text { asthma }}, \frac{47}{\text { sorethroat }}, \frac{43}{\text { cough }}, \frac{38.6}{\text { fever }}, \frac{70}{\text { age }}\right\} .
\end{aligned}
$$

Notice that both $P_{8}$ and $P_{9}$ have two asthma values (we will take their center in the following computation for simplicity) because he/she ate a kind of TCL-like food before taking the two values. As the group of experts give two different weight vectors $\vec{e}=\left(e_{1}, e_{2}, e_{3}, e_{4}, e_{5}\right)$ $=(2,1,5,30,1)$ and $\vec{v}=\left(v_{1}, v_{2}, v_{3}, v_{4}, v_{5}\right)=(4,3,10,60,1)$ for importance of vector of symptom $\vec{s}=($ asthma, sore throat, cough, fever, age), we finish this decision-making problem in the following nine steps (see Figure 1).

Step 1. Compute the difference between $P_{8}$ and those with diagnosis results $\mathrm{Y}$ using a metric rely on $\vec{e}$ (there are many cases on choices of $\left.\rho_{\mathbf{e}}\right) \cdot \rho_{\mathbf{e}}\left(P_{2}, P_{8}\right)=e_{1} \mid 41$ $-61\left|+e_{2}\right| 51-60\left|+e_{3}\right| 41-28\left|+e_{4}\right| 38-37.9\left|+e_{5}\right| 41-67 \mid$ $=143, \rho_{\mathbf{e}}\left(P_{4}, P_{8}\right)=e_{1}|61-45|+e_{2}|60-53|+e_{3}|44-28|$ $+e_{4}|37.8-37.9|+e_{5}|44-67|=145, \rho_{\mathbf{e}}\left(P_{5}, P_{8}\right)=e_{1} \mid 61$ $-39\left|+e_{2}\right| 62-60\left|+e_{3}\right| 49-28\left|+e_{4}\right| 40-37.9\left|+e_{5}\right| 70-67 \mid$ $=217, \rho_{\mathbf{e}}\left(P_{6}, P_{8}\right)=e_{1}|61-55|+e_{2}|55-60|+e_{3}|36-28|$ $+e_{4}|38-37.9|+e_{5}|50-67|=77, \rho_{\mathbf{e}}\left(P_{7}, P_{8}\right)=e_{1}|61-60|$ $+e_{2}|57-60|+e_{3}|37-28|+e_{4}|38.5-37.9|+e_{5}|54-67|$ $=77$. Thus, we obtain the average $d_{8}, e, Y=132.6$.

Step 2. Compute the difference between $P_{8}$ and those with diagnosis results $\mathrm{N}$ using a metric rely on $\vec{e}$. $\rho_{\mathbf{e}}\left(P_{1}, P_{8}\right)=e_{1}|39-61|+e_{2}|48-60|+e_{3}|18-28|$ $+e_{4}|37.8-37.9|+e_{5}|67-67|=109, \rho_{\mathbf{e}}\left(P_{3}, P_{8}\right)=e_{1} \mid 52$ $-61\left|+e_{2}\right| 48-60\left|+e_{3}\right| 32-28\left|+e_{4}\right| 38.5-37.9\left|+e_{5}\right| 44$ $-67 \mid=91$. Thus, we obtain the average $d_{8}$, e, $N=100$.

Step 3. Compute the difference between $P_{9}$ and those with diagnosis results $\mathrm{Y}$ using a metric rely on $\vec{e}$. $\rho_{\mathbf{e}}\left(P_{2}, P_{9}\right)=103, \rho_{\mathbf{e}}\left(P_{4}, P_{9}\right)=71, \rho_{\mathbf{e}}\left(P_{5}, P_{9}\right)=133$,

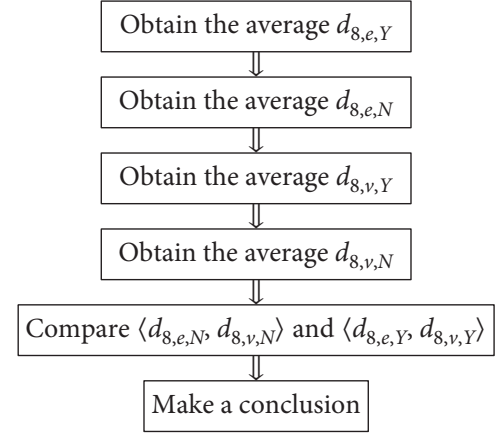

FIgURE 1: A diagram for the proposed algorithm.

$\rho_{\mathbf{e}}\left(P_{6}, P_{9}\right)=95, \rho_{\mathbf{e}}\left(P_{7}, P_{9}\right)=63$. Thus, we obtain an average $\mathrm{d}_{9}, e, Y=93$.

Step 4. Compute the difference between $P_{9}$ and those with diagnosis results $N$ using a metric rely on $\vec{e}$. $\rho_{\mathbf{e}}\left(P_{1}, P_{9}\right)=199, \rho_{\mathbf{e}}\left(P_{2}, P_{9}\right)=105$. Thus, we obtain an average $d_{9}, e, N=152$.

Step 5. Compute the difference between $P_{8}$ and those with diagnosis results $\mathrm{Y}$ using a metric rely on $\vec{v}$. $\rho_{\mathbf{v}}\left(P_{2}, P_{8}\right)=269, \rho_{\mathbf{v}}\left(P_{4}, P_{8}\right)=252, \rho_{\mathbf{v}}\left(P_{5}, P_{8}\right)=433$, $\rho_{\mathbf{v}}\left(P_{6}, P_{8}\right)=128, \rho_{\mathbf{v}}\left(P_{7}, P_{8}\right)=152$. Thus, we obtain an average $d_{8}, v, Y=246.8$.

Step 6. Compute the difference between $P_{8}$ and those with diagnosis results $\mathrm{N}$ using a metric rely on $\vec{v}$. $\rho_{\mathbf{v}}\left(P_{1}, P_{8}\right)=230, \rho_{\mathbf{v}}\left(P_{2}, P_{8}\right)=171$. Thus, we obtain an average $d_{8}, v, N=200.5$.

Step 7. Compute the difference between $P_{9}$ and those with diagnosis results $\mathrm{Y}$ using a metric rely on $\vec{v}$. $\rho_{\mathbf{v}}\left(P_{2}, P_{9}\right)=181, \rho_{\mathbf{v}}\left(P_{4}, P_{9}\right)=146, \rho_{\mathbf{v}}\left(P_{5}, P_{9}\right)=281$, $\rho_{\mathbf{v}}\left(P_{6}, P_{9}\right)=178, \rho_{\mathbf{v}}\left(P_{7}, P_{9}\right)=120$. Thus, we obtain an average $\mathrm{d}_{9}, v, Y=181.2$.

Step 8. Compute the difference between $P_{9}$ and those with diagnosis results $\mathrm{N}$ using a metric rely on $\vec{v}$. $\rho_{\mathbf{v}}\left(P_{1}, P_{9}\right)=396, \rho_{\mathbf{v}}\left(P_{2}, P_{9}\right)=185$. Thus, we obtain an average $d_{9}, v, N=290.5$.

Step 9. As $\left\langle d_{8}\right.$ e $\left., N, d_{8}, v, N\right\rangle=\langle 100,200.5\rangle \leq\langle 132.6$, $246.8\rangle=\left\langle d_{8}, e, Y, d_{8}, v, Y\right\rangle, P_{8}$ is $N$ (which is the same as the diagnosis result given by expert physicians in that hospital); as $\left\langle\mathrm{d}_{9}, e, Y, \mathrm{~d}_{9}, v, Y\right\rangle=\langle 93,181.2\rangle$ $\leq\langle 152,290.5\rangle=\left\langle\mathrm{d}_{9}, e, N, \mathrm{~d}_{9}, v, N\right\rangle, P_{9}$ is $Y$ (which is also the same as the diagnosis result given by expert physicians in that hospital).

\section{Proposition 4}

(1) For a mapping $\rho: X^{2} \longrightarrow \square(R+)$, consider the following conditions:

$$
\begin{aligned}
& (M 0) \rho(x, x)=0(\forall x \in X) \\
& (M 1) \rho(x, y)=0 \text { if } x=y(\forall\{x, y\} \subseteq X) \\
& (M 2) \rho(x, y)=\rho(y, x)(\forall\{x, y\} \subseteq X) \\
& (M 3) \rho(x, y) \leq \rho(x, z) \oplus \rho(z, y)(\forall\{x, y, z\} \subseteq X) \\
& (M 4) p_{2} \circ \rho(x, y) \leq p_{2}[\rho(x, z) \oplus \rho(z, y)]=p_{2} \circ \rho(x, \\
& z)+p_{2} \circ \rho(z, y)(\forall\{x, y, z\} \subseteq X)
\end{aligned}
$$


Then, $\rho$ is a WIVP-metric (resp., an interval-valued pseudo-metric, a weak interval-valued metric, an interval-valued metric) on $X$ if it satisfies $(M 0)+(M 2)+(M 4) \quad($ resp.,$\quad(M 0)+(M 2)+(M 3)$, $(M 1)+(M 2)+(M 4),(M 1)+(M 2)+(M 3))$.

(2) Every interval-valued metric space $(X, \rho)$ can be looked as a special fuzzy metric space, where we define $L$ and $R$ as $L(a, b)=\min \{a, b\}(\forall(a, b) \in[0,1] \times[0,1])$ and $R(a, b)=\max \{a, b\}(\forall(a, b) \in[0,1] \times[0,1])$, and set $d=\rho$.

\section{WIVP-Metrics Pertinent to Logic Theory}

An logic implication operator is a mapping $I:[0,1] \times[0,1]$ $\longrightarrow[0,1]$ which satisfies some conditions (see [41]). There are many well-known logic implication operators (see $[23,24,41])$; some of them are defined as follows:

(i) Boolean implication operator: $I_{B}(a, b)=0$ (if $a=1$, $b=0$ ) or 1 (otherwise)

(ii) Gaines-Rescher implication operator: $I_{G R}(a, b)=1$ (if $a \leq b$ ) or 0 (if $a \leq b$ )

(iii) Goguen implication operator: $I_{G o}(a, b)=1$ (if $a$ $=0)$ or $1 \wedge(b / a)($ if $a \neq 0)$

(iv) Godel implication operator: $I_{G}(a, b)=1$ (if $\left.a \leq b\right)$ or $b$ (if $a \leq b)$

(v) Kleene-Dienes implication operator: $I_{K D}(a, b)=(1$ -a) $\vee b$

(vi) Lukasiewicz implication operator: $I_{L u}(a, b)=(1$ $-a+b) \wedge 1$

(vii) Mamdani implication operator: $I_{M}(a, b)=a \wedge b$

(vii) Product implication operator: $I_{\Pi}(a, b)=1$ (if $a$ $\leq b)$ or $(b / a)$ (if $a \leq b)$

(ix) Wang implication operator: $I_{0}(a, b)=1$ (if $\left.a \leq b\right)$ or $(1-a) \vee b$ (if $a \leq b)$

(x) Reichenbach implication operator: $I_{R}(a, b)=1-a$ $+a b$

(xi) Yager implication operator: $I_{Y}(a, b)=b^{a}(\forall a, b$ $\in[0,1])$

(xii) Zadeh implication operator: $I_{Z}(a, b)=(1-a) \vee(a$ $\wedge b)$

In this section, we will demonstrate how to construct, by using some of these logic implication operators, some WIVP-metrics that may be used in quantitative logic (cf. [23]) and quantitative reasoning (cf. [24]).

\section{Definition 4 (cf. [42])}

(1) Let $S=\left\{p_{1}, p_{2}, \ldots\right\}$ be a countable set (where $p_{i}$ $\neq p_{j}$ if $\left.i \neq j\right)$, a unary operator, $\vee$ and $\longrightarrow$ binary operators, and $F(S)$ the free algebra of type $\{, \vee, \longrightarrow\}$ generated by $S$. Elements of $S$ are called atomic formulae (or atomic propositions), and elements of $F(S)$ are called formulae (or propositions).

(2) $[0,1]$ can be looked as an algebra of type $\{, V$, $\longrightarrow\}$ if we define the unary operator : $[0,1]$ $\longrightarrow[0,1]$ and the two binary operators $\vee:[0,1]$ $\times[0,1] \longrightarrow[0,1]$ and $\longrightarrow:[0,1] \times[0,1] \longrightarrow[0,1]$ as follows: $a=1-a(\forall a \in[0,1]), a \vee b=\max \{a, b\}(\forall a$, $b \in[0,1]), a \longrightarrow b=I(a, b)(\forall a, b \in[0,1])$, where $I:[0,1] \times[0,1] \longrightarrow[0,1]$ is a logic implication operator. Furthermore, for each $A \in F(S)$, there exists a unique finite subset $S_{A} \subseteq F(S)$ (without loss of generality we assume $\left.S_{A}=\left\{p_{1}, p_{2}, \ldots, p_{n}\right\}\right)$ such that $A$ can be represented uniquely by $S_{A}$ and $\{, \vee, \longrightarrow\}$ (written as $\left.A=A\left(p_{1}, p_{2}, \ldots, p_{n}\right)\right)$. Substitute $x_{k}$ for $p_{k}$ in $A\left(p_{1}\right.$, $\left.p_{2}, \ldots, p_{n}\right)(k=1,2, \ldots, n)$ and keep the logic connectives in $A$ unchanged, then we obtain an expression $\overline{A_{I}}\left(x_{1}, x_{2}, \ldots, x_{n}\right)$ and thus an $n$-variable function $\overline{A_{I}}$ $:[0,1]^{n} \longrightarrow[0,1]$ which is called the $I$-function corresponding to $A$. For example, if $A=p_{1} \longrightarrow\left(p_{2} \vee p_{3}\right)$, then $\overline{A_{I}}\left(x_{1}, x_{2}, x_{3}\right)=I\left(1-x_{1}, \max \left\{x_{2}, x_{3}\right\}\right)$.

(3) A mapping $v: F(S) \longrightarrow[0,1]$ which preserves operations , $\vee$ and $\longrightarrow$ is called an $I$-valuation on $F(S)$. The set of all $I$-valuations is written as $\Omega(I)$.

(4)Let $A, B \in F(S)$. If $v(A)=v(B)(\forall v \in \Omega(I))$, then $A$ and $B$ are said to be $I$-logically equivalent.

Lemma 1. If the formulae $A$ and $B$ in $F(S)$ are I-logically equivalent, then $\overline{A_{I}}=\overline{B_{I}}$ (where $I$ is a logic implication operator).

Proof. Without loss of generality, we assume $S_{A}=\left\{p_{1}, p_{2}\right.$, $\left.\ldots, p_{m}\right\}, S_{B}=\left\{p_{1}, p_{2}, \ldots, p_{n}\right\}$, and $m+1=n$. Let $A^{*}=A$ $\wedge(r \longrightarrow r)$ (where $\left.r \in S_{B}-S_{A}\right)$, then $S_{A^{*}}=S_{B}$. For each $\vec{x}$ $=\left(x_{1}, x_{2}, \ldots, x_{n}\right) \in[0,1]^{n}$, there exists an $I$-valuation $\nu_{\vec{x}}: F(S) \longrightarrow[0,1]$ on $F(S)$ which satisfies $\nu_{\vec{x}}\left(p_{i}\right)=x_{i}(i$ $=1,2, \ldots, n)$ since $F(S)$ is a free algebra. As $A$ and $B$ are $R$-logically equivalent, $\overline{A_{I}}(\vec{x})=\overline{A_{I}^{*}}(\vec{x})=\nu_{\vec{x}}\left(A^{*}\right)=\nu_{\vec{x}}$ $(A)=\nu_{\vec{x}}(B)=\overline{B_{I}}(\vec{x})$, which means $\overline{A_{I}}=\overline{B_{I}}$.

\section{Lemma 2}

(1) For any $A, B \in F(S)$, define $A \sim B$ if and only if $A$ and $B$ is $I_{L u}$-logically equivalent. Then, $\sim$ is an equivalence relation on $F(S)$. The equivalence class determined by $A \in F(S)$ is written $[A]$, and the quotient set determined by $\sim$ is written $F(S) / \sim$.

(2) The mapping $d_{\mathbf{n}}: F(S) \times F(S) \longrightarrow[0,+\infty)$, defined by $(\forall\{A, B\} \subseteq F(S)) d_{\mathbf{n}}(A, B)=1-\int \triangle\left[I_{\mathbf{n}}\left(\overline{A_{I_{\mathbf{n}}}}(\vec{x})\right.\right.$, $\left.\left.\overline{B_{I_{\mathrm{n}}}}(\vec{x})\right) \wedge I_{\mathrm{n}}\left(\overline{B_{I_{\mathrm{n}}}}(\vec{x}), \overline{A_{I_{\mathrm{n}}}}(\vec{x})\right)\right] d \vec{x}$, is a pseudometric on $F(S)(\mathbf{n} \in\{L u, G o, G, 0\})$, where $\triangle$ is the integral domain related $(A, B)$.

(3) For each $\varepsilon \in[0,1]$, the mapping $d_{G R}^{\varepsilon}: F(S) \times F(S)$ $\longrightarrow[0,+\infty)$, defined by $1-\varepsilon \int^{-} \Delta\left[I_{G R}\left(\overline{A_{I_{G R}}}(\vec{x})\right.\right.$, $\left.\left.\overline{B_{I_{G R}}}(\vec{x})\right) \wedge I_{G R}\left(\overline{B_{I_{G R}}}(\vec{x}), \overline{A_{I_{G R}}}(\overrightarrow{\vec{x}})\right)\right] d \vec{x} \quad($ if $A \times B)$ or $d_{G R}^{\varepsilon}(A, B)=0$ (if $A \sim B$ ), is a pseudo-metric on $F(S)$. (4) $d_{G R}^{\varepsilon_{1}} \geq d_{G R}^{\varepsilon_{2}}\left(0 \leq \varepsilon_{1}<\varepsilon_{2} \leq 1\right)$. Notice also that $d_{G R}^{0}(A$, $B)=\{0, A \sim B, 1, A \nsim B$. 
(5) $\left\{\rho_{G R}^{\varepsilon}\right\}_{\varepsilon} \in[0,1)$ is a family of metrics on $F(S) /$ which satisfy $\rho_{G R}^{\varepsilon_{1}} \geq \rho_{G R}^{\varepsilon_{2}}\left(\begin{array}{llll}0 & \leq \varepsilon_{1} & <\varepsilon_{2} & <1) \text {, where }\end{array}\right.$ $\rho_{G R}^{\varepsilon}(\varepsilon \in[0,1])$ is defined by $\rho_{G R}^{\varepsilon}([A],[B])=d_{G R}^{\varepsilon}(A$, $B)(\forall A, B \in F(S))$.

(6)(cf. [42]). The mapping $\rho_{\text {Lu }}: F(S) / \sim \times F(S) / \sim$ $\longrightarrow[0,+\infty)$, defined by $\rho_{L u}([A],[B])=d_{L u}(A, B)($ $\forall A, B \in F(S)$ ), is a metric on $F(S) / \sim$.

Remark 1. Lemma 2 (2) is Theorem 6 (without proof) in [41].

\section{Theorem 1}

(1) $\left(F(S), d_{1} \Delta d_{2}\right)$, is a WIVP-metric space $\left(d_{1}, d_{2}\right.$ $\left.\in\left\{d_{G}, d_{G o}, d_{L u}, d_{0}\right\} \cup\left\{d_{G R}^{\varepsilon} \mid \varepsilon \in[0,1]\right\}\right)$

(2) $\left(F(S) / \sim \rho_{1} \Delta \rho_{2}\right)$, is a weak interval-valued metric space $\left(\rho_{1}, \rho_{2} \in\left\{\rho_{\text {Lu }}\right\} \cup\left\{\rho_{G R}^{\varepsilon} \mid \varepsilon \in[0,1]\right\}\right)$

(3) $\left(F(S) / \sim \rho_{1} \Delta \rho_{2}\right)$, is an interval-valued metric space $\left(\rho_{1}, \rho_{2} \in\left\{\rho_{G R}^{\varepsilon} \mid \mathcal{\varepsilon} \in[0,1]\right\}\right)$

Proof. It follows from Lemma 2 (including its proof) and Remarks 1.

\section{Interval-Valued Metrics on Interval-Valued Fuzzy Graphs}

Metric graph theory abounds in applications (e.g., it is applicable in such different areas as location theory, theoretical biology and chemistry, combinatorial optimization, and computational geometry, see [[43], p.99-121] for details). In this section, we extend the notion of metrics on a graph to interval-valued metrics on an interval-valued fuzzy graph (particularly, on a fuzzy graph) and give some related examples.

An $J$-graph (where $J$ is a completely distributive complete lattice with the least element 0$)$ is a triple $G=(V, \sigma, \mu)$ consisting of a nonempty finite set $V$ and a pair of mappings $\sigma: V \longrightarrow J$ and $\mu: V \times V \longrightarrow J$ which satisfies supp $\sigma=V$ and $\mu(x, y)=\mu(y, x) \leq \sigma(x) \wedge \sigma(y)(\forall(x, y) \in V \times V)$. The underlying graph of $G$ is defined as $[G]=(V, E)$, where $E$ $=\{\{x, y\} \subseteq V \mid \mu(x, y)>0\}$. An $J$-graph $G=(V, \sigma, \mu)$ is said to be connected if its underlying graph $[G]=(V, E)$ is connected, i.e., for any 2-element subset $\{x, y\} \subseteq V$, there exists an $m(x, y)$-element subset $\left.\left\{z_{1}, z_{2}, \ldots, z_{m(x}, y\right)\right\} \subseteq V\left(m_{x y}\right.$ $\geq 2)$ such that $x=z_{1}, z_{m}(x, y)=y$, and, $\left\{z_{1}, z_{2}\right\},\left\{z_{2}, z_{3}\right\}$, $\ldots,\left\{z_{m(x, y)}-1, z_{m(x, y)}\right.$, , are all in $E$; the word $P=z_{1}, z_{2}$, $\ldots, z_{m(x, y)}$ is called a path from $x$ to $y$, and the set of all paths from $x$ to $y$ is denoted by $\mathscr{P}_{x y}$.

Definition 5. Let $G=(V, \sigma, \mu)$ be a $[0,1]$-graph (called also fuzzy graph [42]), and $\varepsilon \in[0,1]$. For each $x \in V$, let $\rho-1(x$, $x)=\rho_{\varepsilon}(x, x)=0$. For any $x, y \in V(x \neq y)$, define $\rho-1(x$, $y)$ and $\rho_{\varepsilon}(x, y)$ as follows:

(1)If there is no path from $x$ to $y$, define $\rho-1(x, y)$ $=\rho_{\varepsilon}(x, y)=|V|$ (the cardinality of $V$ ).

(2)If there is a path from $x$ to $y$, define

$$
\begin{aligned}
\rho_{-1}(x, y)= & \wedge\left\{\left[\mu\left(z_{1}, z_{2}\right)+\mu\left(z_{2}, z_{3}\right)+\cdots+\mu\left(z_{m(x, y)-1}, z_{m(x, y)}\right)\right] \wedge \hat{1 \leq i \leq m(x, y)} \sigma\left(z_{i}\right) \mid z_{1} z_{2}, \ldots, z_{m(x, y)} \in \mathscr{P}_{x y}\right\}, \\
\rho_{\varepsilon}(x, y)= & \wedge\left\{\mu\left(z_{1}, z_{2}\right)\left[\varepsilon\left(\sigma\left(z_{1}\right) \vee \sigma\left(z_{2}\right)\right)+(1-\varepsilon)\left(\sigma\left(z_{1}\right) \wedge \sigma\left(z_{2}\right)\right)\right]+\mu\left(z_{2}, z_{3}\right)\left[\varepsilon\left(\sigma\left(z_{2}\right) \vee \sigma\left(z_{3}\right)\right)+(1-\varepsilon)\left(\sigma\left(z_{2}\right) \wedge \sigma\left(z_{3}\right)\right)\right]\right. \\
& +\cdots+\mu\left(z_{m(x, y)-1}, z_{m(x, y)}\right)\left[\varepsilon\left(\sigma\left(z_{m(x, y)-1}\right) \vee \sigma\left(z_{m(x, y)}\right)\right)+(1-\varepsilon)\left(\sigma\left(z_{m(x, y)-1}\right) \wedge \sigma\left(z_{m(x, y)}\right)\right] \mid z_{1}, z_{2}, \ldots, z_{m(x, y)} \in \mathscr{P}_{x y}\right\} .
\end{aligned}
$$

Then, the mappings $\rho-1, \rho_{\varepsilon}: V \times V \longrightarrow[0,+\infty)$ are metrics on $V$ (called metrics on the fuzzy graph $G$ ).

Remark 2. It is easy to verify $\rho_{\varepsilon_{1}} \leq \rho_{\varepsilon_{2}}$ for all $\varepsilon_{1}, \varepsilon_{2} \in\{-1\}$ $\cup[0,1]$ satisfying $\varepsilon_{1} \leq \varepsilon_{2}$, thus we obtain a family $\left\{\rho_{\varepsilon_{1}} \Delta \rho_{\varepsilon_{2}} \mid \varepsilon_{1}\right.$ $\left.\leq \varepsilon_{2}, \varepsilon_{1}, \varepsilon_{2} \in\{-1\} \cup[0,1]\right\}$ of interval-valued metrics on $V$ (called interval-valued metrics on the fuzzy graph $G$ ).

Theorem 2. Let $G=(V, \sigma, \mu)$ be an $\square([0,1])$-graph (called also interval-valued fuzzy graph). For any $x, y \in V$, let

$$
\rho(x, y)= \begin{cases}0, & x=y, \\ \wedge\left\{\ell(P) \mid P \in \mathscr{P}_{x y}\right\}, & x \neq y \text { and } \mathscr{P}_{x y} \neq \varnothing, \\ \left\langle\frac{|V|^{2}}{m(\mu)}, \frac{|V|^{2}}{m(\mu)}\right\rangle, & x \neq y \text { and } \mathscr{P}_{x y}=\varnothing,\end{cases}
$$

where 


$$
\begin{aligned}
& \ell(P)=\frac{1}{\mu\left(z_{1}, z_{2}\right)}+\frac{1}{\mu\left(z_{2}, z_{3}\right)}+\cdots+\frac{1}{\mu\left(z_{n-1}, z_{n}\right)}, \quad\left(\forall P=z_{1}, z_{2}, \ldots, z_{n} \in \mathscr{P}_{x y}\right), \\
& \frac{1}{\langle\dot{a}, a\rangle}=\left\langle\frac{1}{\dot{a} \wedge} \wedge \frac{1}{a}, \frac{1}{\dot{a}} \vee \frac{1}{a}\right\rangle, \quad(\forall\langle\dot{a}, a\rangle \in \mathbb{Q}((0,+\infty))),
\end{aligned}
$$

and $m(\mu)=\min \{\mu(s, t) \mid\{s, t\} \in E\}$. Then, $\rho$ is an intervalvalued metric on $V$ (called interval-valued metric on the interval-valued fuzzy graph $G$ ).

\section{Fixed Point Theorems and Common Fixed Point Theorems in WIVP-Metrics}

\section{Definition 6}

(1) A sequence $\left\{x_{n}\right\}$ in a WIVP-metric space $(X, \rho)$ is said to be a Cauchy sequence if, for each $\langle\varepsilon-, \varepsilon+\rangle \gg 0$, there exists a positive integer $n_{\varepsilon}$ such that $\rho\left(x_{n}, x_{m}\right)$ $\ll\langle\varepsilon-, \varepsilon+\rangle\left(\forall n, m \geq n_{\varepsilon}\right) ;\left\{x_{n}\right\}$ is said to be convergent in $(X, \rho)$ if there exists an $x \in X$ such that $\left\{x_{n}\right\}$ converges to $x$ (i.e., for each $\langle\varepsilon-, \varepsilon+\rangle \gg 0$, there exists a positive integer $n_{\varepsilon}$ such that $\rho\left(x_{n}, x\right) \ll\langle\varepsilon-, \varepsilon+\rangle$ ( $\left.\forall n \geq n_{\varepsilon}\right)$.

(2) A WIVP-metric space $(X, \rho)$ is said to be complete if every Cauchy sequence in $(X, \rho)$ is convergent.

It is not difficult to verify the following.

Lemma 3. A WIVP-metric space $(X, \rho)$ is complete if and only if $(X, \rho+)$ is complete.

The main results of this section are the following.

Theorem 3. Let $(X, \rho)$ be a complete WIVP-metric space. Then, $f: X \longrightarrow X$ has a unique fixed point in $X$ if it satisfies one of the following conditions:

(1) $\rho+(f(x), f(y)) \leq k \rho+(x, y)(\forall x, y \in X)$, where $k$ $\in[0,1)$ is a constant.

(2) $\rho+(f(x), f(y)) \leq k[\rho+(x, f(x))+\rho+(y$, $f(y))](\forall x, y \in X)$, where $k \in(0,(1 / 2))$ is a constant.

(3) $\rho+(f(x), f(y)) \leq k \max \{\rho+(x, f(x)), \rho+(y$, $f(y))\}(\forall x, y \in X)$, where $k \in[0,1)$ is a constant.

(4) $\rho+(f(x), f(y)) \leq a \rho+(x, f(x))+b \rho+(y, f(y))$ $+c \rho+(x, y)(\forall x, y \in X)$, where $a, b, c$ are nonnegative numbers satisfying $a+b+c<1$.

(5) $\rho+(f(x), f(y)) \leq k[\rho+(x, f(y))+\rho+(y$, $f(x))](\forall x, y \in X)$, where $k \in(0,(1 / 2))$ is a constant.

(6) $\rho+(f(x), f(y)) \leq k \max \{\rho+(x, f(y)), \rho+(y$, $f(x))\}(\forall x, y \in X)$, where $k \in[0,(1 / 2))$ is a constant.

(7) $\rho+(f(x), f(y)) \leq a \rho+(x, f(y))+b \rho+(y, f(x))$ $+c \rho+(x, y)(\forall x, y \in X)$, where $a, b, c$ are nonnegative numbers satisfying $a+b+c<1$.

(8) $\rho+(f(x), f(y)) \leq k_{1} \rho+(x, f(x))+k_{2} \rho+(y, f(y))$ $+k_{3} \rho+(x, f(y))+k_{4} \rho+(y, f(x))+k_{5} \rho+(x, y),(\forall x$, $y \in X)$, where $k_{i} \geq 0(i \in\{1,2, \ldots, 5\}), \sum_{i}=1^{5} k_{i}<1$, and $s$ is a fixed positive integer.

(9) $\rho+\left(f^{s}(x), f^{s}(y)\right) \leq k_{1} \rho+\left(x, f^{s}(x)\right)+k_{2} \rho+(y$, $\left.f^{s}(y)\right)+k_{3} \rho+\left(x, f^{s}(y)\right)+k_{4} \rho+\left(y, f^{s}(x)\right)+k_{5} \rho+(x$, $y),(\forall x, y \in X)$ where $k_{i} \geq 0(i \in\{1,2, \ldots, 5\}), \sum_{i}$ $=1^{5} k_{i}<1$, and $s$ is a fixed positive integer.

Theorem 4. Let $(X, \rho)$ be a complete weak interval-valued metric space. Then, $f, g: X \longrightarrow X$ have common fixed point in $X$ if they satisfy one of the following conditions (where s and $t$ are fixed positive integers):

(1) $\rho+\left(f^{s}(x), g^{t}(y)\right) \leq k \rho+(x, y)(\forall x, y \in X)$, where $k \in[0,1)$ is a constant.

(2) $\rho+\left(f^{s}(x), g^{t}(y)\right) \leq k\left[\rho+\left(x, f^{s}(x)\right)+\rho+(y\right.$, $\left.\left.g^{t}(y)\right)\right](\forall x, y \in X)$, where $k \in(0,(1 / 2))$ is a constant

(3) $\rho+\left(f^{s}(x), g^{t}(y)\right) \leq k \max \left\{\rho+\left(x, f^{s}(x)\right), \rho+(y\right.$, $\left.\left.g^{t}(y)\right)\right\}(\forall x, y \in X)$, where $k \in[0,1)$ is a constant.

(4) $\rho+\left(f^{s}(x), g^{t}(y)\right) \leq a \rho+\left(x, f^{s}(x)\right)+b \rho+(y$, $\left.g^{t}(y)\right)+c \rho+(x, y)(\forall x, y \in X)$, where $a, b, c$ are nonnegative numbers satisfying $a+b+c<1$.

(5) $\rho+\left(f^{s}(x), g^{t}(y)\right) \leq k\left[\rho+\left(x, g^{t}(y)\right)+\rho+(y\right.$, $\left.\left.f^{s}(x)\right)\right](\forall x, y \in X)$, where $k \in(0,(1 / 2))$ is a constant.

(6) $\rho+\left(f^{s}(x), g^{t}(y)\right) \leq k \max \left\{\rho+\left(x, g^{t}(y)\right), \rho+(y\right.$, $\left.\left.f^{s}(x)\right)\right\}(\forall x, y \in X)$, where $k \in[0,(1 / 2))$ is a constant.

(7) $\rho+\left(f^{s}(x), g^{t}(y)\right) \leq k_{1} \rho+\left(x, f^{s}(x)\right)+k_{2} \rho+(y$, $\left.g^{t}(y)\right)+k_{3} \rho+\left(x, g^{t}(y)\right)+k_{4} \rho+\left(y, f^{s}(x)\right)+k_{5} \rho+(x$, $y)(\forall x, y \in X)$, where $k_{i} \geq 0 i \in\{1,2, \ldots, 5\}, \sum_{i}=1{ }^{5} k_{i}$ $<1$.

Theorem 3 may be proved based on Lemma 3 and results on contractive-type mappings satisfying (1), (4), (5), (7), (11), (12), (14), (18), (43) in [44], and Theorem 4 may be proved based on Lemma 3 and results on contractive-type mappings satisfying (176), (179), (180), (182), (186), (187), (193) in [44].

\section{Concluding Remarks}

Since data from many real-world problems are not only from multi agents but also becoming more and more big and complex for vagueness and uncertainty, measurement by a single metric does not meet the needs of some practical problems. Motivated by Polya's plausible reasoning and artificial neural networks, this paper consider a distance-like 
notion, called weak interval-valued pseudo-metric (WIVPmetric for short), which, as a generalization of the notion of metric, is still topologically good. To benefit practitioners, easy-to-understand propositions and much detailed examples are given (in the first half of the paper) to illustrate how to fabricate (including using what "material") an expected or demanded WIVP-metric (even interval-valued metric) in practical problems. To show theoretical applications of WIVP-metrics, we exemplify how to construct (by using some logic implication operators, some WIVP-metrics which may be useful in quantitative logic [23] and quantitative reasoning [24]) and how to define well-matched interval-valued metrics on interval-valued fuzzy graphs. As these WIVP-metrics are relatively precise, flexible, and compatible than single pseudo-semi-metric, pseudo-metric, and metric, more applications should be investigated (even put forward) based on plausible reasoning. Practitioners are also suggested to explore (in the plausible reasoning manner) other complex and more fitted methods to fabricate more desired distance-like measures, for example, to fuse easy-to-obtain pseudo-semi-metrics, pseudo-metrics, or metrics by making full use of well-known or frequently used t-norms, t-conorms, aggregation operators, and similar operators coined by practitioners or others; strategies also contain making full use of interval numbers and very special triangular fuzzy numbers. Of course, these (including also determination of weighted vector) are not always easy to practitioners. How to overcome this limitation will be one of our future works.

Our future work also includes completion of WIVPmetric spaces, interval-valued truth degrees of formulas based on deferent logic implication operators, intervalvalued similarity degrees of formulas based on deferent logic implication operators, related approximate reasoning, dynamic systems on interval-valued metric spaces (even on interval-valued pseudo-metric spaces), and applications of weak interval-valued pseudo-metrics in medical diagnosis and decision-making problems (see related works [45-51] for details); for decision-making problems with data given by a fuzzy set (resp., interval-valued fuzzy sets, picture fuzzy sets, generalized pythagorean fuzzy sets), one can use the WIVP-metrics used in Example 2 to replace distance measures used in published papers and use the linear orders in $[32,33,52]$ to replace the ordinary order in the real line.

\section{Appendix}

Proof. of Proposition 1. We only prove $\rho_{3}(\vec{a}, \vec{b}) \leq \rho_{4}(\vec{a}$, $\vec{b})$ conditionally. If $\vec{a}=\vec{b}$, then $\rho_{3}(\vec{a}, \vec{b})=0=\rho_{4}(\vec{a}$, $\vec{b})$. Next, we assume $(\vec{a}, \vec{b})$ satisfies $a_{1} \neq b_{1}$ and $\mid\left(a_{2}\right.$ $\left.\left.-b_{2}\right)\left(a_{3}-b_{3}\right) \mid-a_{2} b_{2}-a_{3} b_{3} \leq \sqrt{\left(a_{2}^{2}\right.}+a_{3}^{2}\right)\left(b_{2}^{2}+b_{3}^{2}\right)$. There are only four cases which need to be considered.

Case $1\left(a_{2}-b_{2}\right)\left(a_{3}-b_{3}\right)=0$. Then, $\rho_{3}(\vec{a}, \vec{b})-\mid a_{1}$ $\left.-b_{1} \mid=\sqrt{\left(a_{2}\right.}-b_{2}\right)^{2}+\left(a_{3}-b_{3}\right)^{2} \leq \sqrt{a_{2}^{2}}+a_{3}^{2}+\sqrt{b_{2}^{2}}+b_{3}^{2}$ $\underset{\vec{b}}{\leq \rho_{4}}(\vec{a}, \vec{b})-\left|a_{1}-b_{1}\right|$, and thus $\rho_{3}(\vec{a}, \vec{b}) \leq \rho_{4}(\vec{a}$, $\vec{b}$ ).

Case $2\left(a_{2}-b_{2}\right)\left(a_{3}-b_{3}\right) \neq 0$ and $\left(b_{3} / a_{3}\right)=\left(b_{2} / a_{2}\right)=k$ $>0$. As $\left|a_{2} a_{3}\right|\left(k^{2}-2 k+1\right) \leq 2 k\left(a_{2}^{2}+a_{3}^{2}\right), \rho_{3}(\vec{a}, \vec{b})$ $-\left|a_{1}-b_{1}\right|=|k-1|\left(\left|a_{2}\right|+\left|a_{3}\right|\right) \leq(k+1) \sqrt{a_{2}^{2}}+a_{3}^{2}=\rho_{4}(\vec{a}$ $, \vec{b})-\left|a_{1}-b_{1}\right|$, and thus $\rho_{3}(\vec{a}, \vec{b}) \leq \rho_{4}(\vec{a}, \vec{b})$. Since $\left.\left|\left(a_{2}-b_{2}\right)\left(a_{3}-b_{3}\right)\right|-a_{2} b_{2}-a_{3} b_{3} \leq \sqrt{\left(a_{2}^{2}\right.}+a_{3}^{2}\right)\left(b_{2}^{2}+b_{3}^{2}\right)$, the case that $\left(a_{2}-b_{2}\right)\left(a_{3}-b_{3}\right) \neq 0$ and $\left(b_{3} / a_{3}\right)=\left(b_{2}\right.$ $\left./ a_{2}\right) \leq 0$ cannot appear.

Case $3\left(a_{2}-b_{2}\right)\left(a_{3}-b_{3}\right) \neq 0$ and $\left(b_{3} / a_{3}\right) \neq\left(b_{2} / a_{2}\right)$. Write $O=(0,0), A=\left(a_{2}, a_{3}\right), B=\left(b_{2}, b_{3}\right), T=\left(a_{2}, b_{3}\right)$. Then, $\rho_{3}(\vec{a}, \vec{b})-\left|a_{1}-b_{1}\right|=\left|a_{2}-b_{2}\right|+\left|a_{3}-b_{3}\right|=\sqrt{\left(a_{2}\right.}$ $\left.\left.-a_{2}\right)^{2}+\left(a_{3}-b_{3}\right)^{2}+\sqrt{\left(b_{2}\right.}-a_{2}\right)^{2}+\left(b_{3}-b_{3}\right)^{2}>0, \rho_{4}(\vec{a}$, $\vec{b})-\left|a_{1}-b_{1}\right|=\sqrt{a_{2}^{2}}+a_{3}^{2}+\sqrt{b_{2}^{2}}+b_{3}^{2}>0$. Therefore, $\left(\rho_{3}(\vec{a}, \vec{b})-\left|a_{1}-b_{1}\right|\right)^{2}=|A T|^{2}+|B T|^{2}+2|A T \| B T|$ $=|A B|^{2}+2|A T \| B T|=|A B|^{2}+2 h_{T}|A B|=|A B|^{2}+2 \mid\left(a_{2}\right.$ $\left.-b_{2}\right)\left(a_{3}-b_{3}\right) \mid$ and $\left(\rho_{4}(\vec{a}, \vec{b})-\left|a_{1}-b_{1}\right|\right)^{2}=|O A|^{2}$ $+|O B|^{2}+2\left|O A\left\|\left.O B|=| A B\right|^{2}+2|O A \| O B|(1+\cos \right.\right.$ $\angle A O B)=|A B|^{2}, \quad+h_{O}|A B|((1+\cos \angle A O B) / \sin$ $\angle A O B)=|A B|^{2}+\left(|O A|^{2}+|O B|^{2}-|A B|^{2}\right)=|A B|^{2}$ $\left.+2\left(\sqrt{\left(a_{2}^{2}\right.}+a_{3}^{2}\right)\left(b_{2}^{2}+b_{3}^{2}\right)+a_{2} b_{2}+a_{3} b_{3}\right)>0$, where $h_{O}$ and $h_{T}$ are the heights of triangles $A O B$ and $A T B$ (with $A B$ as the common base), respectively. As $\mid\left(a_{2}-b_{2}\right)\left(a_{3}\right.$ $\left.\left.-b_{3}\right) \mid-a_{2} b_{2},-a_{3} b_{3} \leq \sqrt{\left(a_{2}^{2}\right.}+a_{3}^{2}\right)\left(b_{2}^{2}+b_{3}^{2}\right), \rho_{3}(\vec{a}, \vec{b})$ $\leq \rho_{4}(\vec{a}, \vec{b})$.

Case $4\left(a_{2}-b_{2}\right)\left(a_{3}-b_{3}\right) \neq 0$ and $a_{2} a_{3}=0$. Without loss of generality, we assume $a_{2}=0$. If $b_{3}=0$, then $\rho_{3}(\vec{a}$, $\vec{b})-\left|a_{1}-b_{1}\right|=\left|b_{2}\right|+\left|a_{3}\right|=\rho_{4}(\vec{a}, \vec{b})-\left|a_{1}-b_{1}\right|$. Thus, $\rho_{3}(\vec{a}, \vec{b})=\rho_{4}(\vec{a}, \vec{b})$. If $b_{3} \neq 0$, then from the proof of Case 3 we can see $\rho_{3}(\vec{a}, \vec{b}) \leq \rho_{4}(\vec{a}, \vec{b})$.

Proof. of Proposition 4. We only prove (1). Firstly, we prove $\rho$ satisfies condition (2) (i) of Definition 1. By (M3) and Definition $1, \lambda_{a}(x, y)=\lambda_{1}(x, y)=\rho-(x, y) \leq \rho-(x, z)+\rho$ $-(z, y)=\lambda_{1}(x, z)+\lambda_{1}(z, y)=\lambda_{a}(x, z)+\lambda_{a}(z, y)(\forall x, y, z$ $\in X, \forall a \in(0,1])$. Write $a=\mathrm{d}(x, z)(s), b=\mathrm{d}(z, y)(t)$, and $c=\min \{a, b\}$. Then, $c \in\{0,1\}$. It does not loose generality to assume $c=1$ (i.e., $a=b=1)$, then $\lambda_{a}(x, z)=\lambda_{1}(x, z)=\rho$ $-(x, z) \leq s$ and $\lambda_{b}(z, y)=\lambda_{1}(z, y)=\rho-(z, y) \leq t$. Since $\lambda_{r}$ is nondecreasing with $r$ and $s+t \leq \lambda_{1}(x, y), \lambda_{1}(x, y)=\lambda_{c}(x$, $y) \leq \lambda_{c}(x, z)+\lambda_{c}(z, y) \leq \lambda_{a}(x, z)+\lambda_{b}(z, y) \leq s+t \leq \lambda_{1}(x$, y) (i.e., $\left.s+t=\lambda_{1}(x, y)=\rho-(x, y)\right)$. Hence, $d(x, y)(s+t)$ $=1 \geq 1=\min (d(x, z)(s), d(z, y)(t))=L(d(x, z)(s), d(z$, y) $(t))$.

Now we prove $\rho$ satisfies condition (2) (ii) of Definition 1. By (M3) and Definition 3, $v_{a}(x, y)=v_{1}(x, y)=\rho+(x, y)$ $\leq \rho+(x, z)+\rho+(z, y)=v_{1}(x, z)+v_{1}(z, y)=v_{a}(x, z)$ $+v_{a}(z, y)(\forall x, y, z \in X, \forall a \in(0,1])$. Write $a=d(x, z)(s$ $+t)$. If $s \geq \lambda_{1}(x, z), t \geq \lambda_{1}(z, y)$, and $s+t \geq \lambda_{1}(x, y)$. Then, $s$ 
$+t \leq v_{a}(x, y)=v_{1}(x, y) \leq \nu_{1}(x, z)+v_{1}(z, y)$. Hence, $s$ $\leq v_{1}(x, z)$ or $t \leq \nu_{1}(z, y)$. This implies that $d(x, z)(s) \geq 1$ or $d(z, y)(t) \geq 1$ and thus $R(d(x, z)(s), d(z, y)(t))$ $=\max \{d(x, z)(s), d(z, y)(t)\}=1 \geq d(x, y)(s+t)$.

Proof. of Lemma 2. We only prove (2) (for the case of $\mathbf{n}$ $=L u)$ and (3). Write $\xi(A, B)=1-d_{L u}(A, B)$. As $1-\overline{A_{I_{L u}}}(\vec{x})$ $+\overline{B_{I_{L u}}}(\vec{x}) \leq 1$ or $1-\overline{B_{I_{L u}}}(\vec{x})+\overline{A_{I_{L u}}}(\vec{x}) \leq 1, \xi(A, B)=\int$ $\triangle\left[\left(1-\overline{A_{I_{L u}}}(\vec{x})+\overline{B_{I_{L u}}}(\vec{x})\right) \wedge\left(1-\overline{{I_{I u}}_{L u}}(\vec{x})+\overline{A_{I_{L u}}}(\vec{x})\right)\right] \mathrm{d} \vec{x}$.

We first show that $d_{L u}(A, B)=0$ if and only if $A$ and $B$ are $I_{L u}$-logically equivalent (particularly, $d_{L u}(A, A)=0$ for all $A \in F(S)$ ). Actually, if $\mathrm{d}_{L u}(A, B)=0$ (i.e., $\xi(A, B)=1$ ), then $1-\overline{A_{I_{L u}}}(\vec{x})+\overline{B_{I_{L u}}}(\vec{x})=1-\overline{B_{I_{L u}}}(\vec{x})+\overline{A_{I_{L u}}}(\vec{x})=1($ $\forall x \in \triangle)$ since $I_{L u}$ is continuous. Thus, $v(A \longrightarrow B)=v(A)$ $\longrightarrow \nu(B)=(1-\nu(A)+\nu(B)) \wedge 1=1$ and $\nu(B \longrightarrow A)=$ $\nu(B) \longrightarrow \nu(A)=(1-\nu(B)+\nu(A)) \wedge 1=1\left(\forall \nu \in \Omega\left(I_{L u}\right)\right)$. It follows that $\nu(A) \leq \nu(B)$ and $\nu(B) \leq \nu(A)$, i.e., $\nu(A)=\nu(B)$. Since $v$ is arbitrary, $A$ and $B$ are $I_{L u}$-logically equivalent.
Conversely, if $A$ and $B$ are $I_{L u}$-logically equivalent, then $\overline{A_{I_{L u}}}$ $=\overline{B_{I_{L u}}}$ (and thus $\left.d_{L u}(A, B)=0\right)$ by Lemma 1. $d_{L u}(A, B) \stackrel{I_{L u}}{=}$ $d_{L u}(B, A)(\forall A, B \in F(S))$ follows from definition of $d_{L u}$. It remains to prove the triangle inequality.

Step 1. $f(a, b)+f(b, c) \leq f(a, c)+1$, where $f(s, t)$ $=I_{L u}(s, t) \wedge I_{L u}(t, s)$. It is not loss of generality to assume $a \geq c$. We consider the following three cases.

(i) $a \leq b$. Then, $f(a, b)=1, f(b, c)=1-b+c$, and thus $f(a, b)+f(b, c)=2-b+c \leq 2-a+c=f(a, c)+1$. (ii) $c \leq b<a$. Then, $f(a, b)=1-a+b, f(b, c)=1-b$ $+c$, and thus $f(a, b)+f(b, c)=2-a+c=f(a, c)+1$. (iii) $b<c$. Then, $f(a, b)=1-a+b, f(b, c)=1$, and thus $f(a, b)+f(b, c)=2-a+b<2-a+c=f(a, c)$ +1 .

Step 2. By the inequality in Step 1, we have $(\{A, B, C\}$ $\subseteq F(S)$ )

$$
\begin{aligned}
d_{L u}(A, B) & =1-\int_{\Delta} f\left(\overline{A_{I_{L u}}}(\vec{x}), \overline{B_{I_{L u}}}(\vec{x})\right) \mathrm{d} \vec{x} \leq 1-\int_{\Delta}\left[f\left(\overline{A_{I_{L u}}}(\vec{x}), \overline{C_{I_{L u}}}(\vec{x})\right)+f\left(\overline{C_{I_{L u}}}(\vec{x}), \overline{B_{I_{L u}}}(\vec{x})\right)-1\right] \mathrm{d} \vec{x} \\
& =\left(1-\int_{\Delta} f\left(\overline{A_{I_{L u}}}(\vec{x}), \overline{C_{I_{L u}}}(\vec{x})\right) \mathrm{d} \vec{x}\right)+\left(1-\int_{\Delta} f\left(\overline{C_{I_{L u}}}(\vec{x}), \overline{B_{I_{L u}}}(\vec{x})\right) \mathrm{d} \vec{x}\right)=d_{L u}(A, C)+d_{L u}(C, B) .
\end{aligned}
$$

(3) By definition of $d_{G R}^{\varepsilon}$ and Lemma 1, we know that $\mathrm{d}_{G R}^{1}(A, B)=0$ if and only if $\overline{A_{I_{G R}}}(\vec{x})=\overline{B_{I_{G R}}}(\vec{x})$ (a.e), and that $d_{G R}^{\varepsilon}(A, B)=0$ if and only if $A$ and $A$ are $I_{G R}$-logically equivalent $(\varepsilon \in[0,1))$. Particularly, $d_{G R}^{\varepsilon}(A, A)=0(\forall A \in F(S))$ holds. Since $d_{G R}^{\varepsilon}(A, B)$ $=d_{G R}^{\varepsilon}(B, A)(\forall A, B \in F(S)), \forall \varepsilon \in[0,1]$ is obvious, we only need to prove the triangle inequality. It is not loss of generality to consider only the case $\varepsilon \in(0,1]$. Step 1. $f(a, b)+f(b, c) \leq f(a, c)+1$, where $f(s, t)$ $=I_{G R}(s, t) \wedge I_{G R}(t, s)$. It is not loss of generality to assume $a<c$. Then, $f(a, c)=I_{G R}(a, c) \wedge I_{G R}(c, a)=1$ $\wedge 0=0$, thus we only need to prove $f(a, b)+f(b, c) \leq 1$. We consider the following five cases. (i) $b<a$. As $f(a, b)=I_{G R}(a, b) \wedge I_{G R}(b, a)=0 \wedge 1=0$, $f(a, b)+f(b, c) \leq 1$

(ii) $b=a$. As $f(b, c)=I_{G R}(b, c) \wedge I_{G R}(c, b)=1 \wedge 0=0$, $f(a, b)+f(b, c) \leq 1$

(iii) $a<b<c$. As $f(a, b)=I_{G R}(a, b) \wedge I_{G R}(b, a)=1$ $\wedge 0=0, f(a, b)+f(b, c) \leq 1$

(iv) $b=c$. As $f(a, b)=I_{G R}(a, b) \wedge I_{G R}(b, a)=1 \wedge 0$ $=0, f(a, b)+f(b, c) \leq 1$

(v) $c<b$. As $f(a, b)=I_{G R}(a, b) \wedge I_{G R}(b, a)=1 \wedge 0=0$, $f(a, b)+f(b, c) \leq 1$

Step 2. For the case $\varepsilon=1$, by Lemma 1 and the inequality in Step 1, we have $(\{A, B, C\} \subseteq F(S))$

$$
\begin{aligned}
d_{G R}^{\varepsilon} & =1-\int_{\Delta} f\left(\overline{A_{I_{G R}}}(\vec{x}), \overline{B_{I_{G R}}}(\vec{x})\right) \mathrm{d} \vec{x} \leq 1-\int_{\Delta}\left[f\left(\overline{A_{I_{G R}}}(\vec{x}), \overline{C_{I_{G R}}}(\vec{x})\right)+f\left(\overline{C_{I_{G R}}}(\vec{x}), \overline{B_{I_{G R}}}(\vec{x})\right)-1\right] \mathrm{d} \vec{x} \\
& =\left(1-\int_{\Delta} f\left(\overline{A_{I_{G R}}}(\vec{x}), \overline{C_{I_{G R}}}(\vec{x})\right) \mathrm{d} \vec{x}\right)+\left(1-\int_{\Delta} f\left(\overline{C_{I_{G R}}}(\vec{x}), \overline{B_{I_{G R}}}(\vec{x})\right) \mathrm{d} \vec{x}\right)=\mathrm{d}_{G R}^{\varepsilon}(A, C)+\mathrm{d}_{G R}^{\varepsilon}(C, B) .
\end{aligned}
$$

For the case $\varepsilon \in(0,1)$, take $A, B, C \in F(S)$. We consider the following two cases (it is not loss of generality to assume $A$ and $B$ are not $I_{G R}$-logically equivalent). (i) Neither $A$ and $C$ are $I_{G R}$-logically equivalent nor $C$ and $B$ are $I_{G R}$-logically equivalent. By the inequality in Step 1, we have 


$$
\begin{aligned}
d_{G R}^{\varepsilon}(A, B) & =1-\varepsilon \int_{\triangle} f\left(\overline{A_{I_{G R}}}(\vec{x}), \overline{B_{I_{G R}}}(\vec{x})\right) \mathrm{d} \vec{x} \leq 1-\varepsilon \int_{\triangle}\left[f\left(\overline{A_{I_{G R}}}(\vec{x}), \overline{C_{I_{G R}}}(\vec{x})\right)+f\left(\overline{C_{I_{G R}}}(\vec{x}), \overline{B_{I_{G R}}}(\vec{x})\right)-1\right] \mathrm{d} \vec{x} \\
& \leq\left(1-\varepsilon \int_{\triangle} f\left(\overline{A_{I_{G R}}}(\vec{x}), \overline{C_{I_{G R}}}(\vec{x})\right) \mathrm{d} \vec{x}\right)+\left(1-\varepsilon \int_{\triangle} f\left(\overline{C_{I_{G R}}}(\vec{x}), \overline{B_{I_{G R}}}(\vec{x})\right) \mathrm{d} \vec{x}\right)=d_{G R}^{\varepsilon}(A, C)+d_{G R}^{\varepsilon}(C, B)
\end{aligned}
$$

(ii) Either $A$ and $C$ are $I_{G R}$-logically equivalent or $C$ and $B$ are $I_{G R}$-logically equivalent. It is not loss of generality to assume that $A$ and $C$ are $I_{G R}$-logically equivalent). By Lemma 1 , we have $d_{G R}^{\varepsilon}(A, B)=d_{G R}^{\varepsilon}(C, B) \leq d_{G R}^{\varepsilon}(A, C)$ $+d_{G R}^{\varepsilon}(C, B)$.

Proof. of Theorem 2We only show that $\rho$ satisfies (M3) in Remark 1. Suppose $\{x, y, z\}$ is a 3-element subset of $V$. If $\mathscr{P}_{x z}=\varnothing$, then $\mathscr{P}_{x y}=\varnothing$ or $\mathscr{P}_{y z}=\varnothing$, and thus $\rho(x, z) \leq \rho(x$, $y) \oplus \rho(y, z)$ (which means that $\rho$ satisfies (M3)). If $\mathscr{P}_{x z} \neq \varnothing$, then we consider the following two cases:

Case $1 \mathscr{P}_{x y}=\varnothing$ or $\mathscr{P}_{y z}=\varnothing$. Then $\rho(x, z) \leq\left\langle\left(|V|^{2}\right.\right.$ $\left.\mid m(\mu)), \quad\left(|V|^{2} \mid m(\mu)\right)\right\rangle \leq \rho(x, y) \oplus \rho(y, z)$, which means that $\rho$ satisfies (M3).

Case $2 \mathscr{P}_{x y} \neq \varnothing$ and $\mathscr{P}_{y z} \neq \varnothing$. For each path $P_{1}=u_{1}$, $u_{2}, \ldots, u_{m} \in \mathscr{P}_{x y}$ from $x$ to $y$ and each path $P_{2}=v_{1} v$, ${ }_{2}, \ldots, v_{n} \in \mathscr{P}_{y z}$ from $y$ to $z$, there exists a path $P_{1,2}$ $=w_{1}, w_{2}, \ldots, w_{l} \in \mathscr{P}_{x z}$ from $x$ to $z$ such that $\left\{w_{1}, w_{2}\right.$, $\left.\ldots, w_{l}\right\} \subseteq\left\{u_{1}, u_{2}, \ldots, u_{m}\right\} \cup\left\{v_{1}, v_{2}, \ldots, v_{n}\right\}$. Therefore, $\rho(x, z)=\wedge\left\{\ell(P) \mid P \in \mathscr{P}_{x z}\right\} \leq \wedge\left\{\ell\left(P_{1,2}\right) \mid P_{1} \in \mathscr{P}_{x y}\right.$, $\left.P_{2} \in \mathscr{P}_{y z}\right\} \leq \wedge\left\{\ell\left(P_{1}\right) \mid P_{1} \in \mathscr{P}_{x y}\right\} \oplus \wedge\left\{\ell\left(P_{2}\right) \mid P_{2} \in \mathscr{P}_{y z}^{x y}\right\}$ $=\rho(x, y) \oplus \rho(y, z)$, which means that $\rho$ satisfies (M3).

\section{Data Availability}

The data used to support the findings of this study are included within the article.

\section{Conflicts of Interest}

The authors declare that there are no conflicts of interest regarding the publication of this paper.

\section{Acknowledgments}

The work was supported by the National Natural Science Foundation of China (Grant nos. 11771263, 61967001, and 61807023) and the Fundamental Research Funds for the Central Universities (Grant nos. GK202105007 and GK201702011).

\section{References}

[1] M. A. Erceg, "Metric spaces in fuzzy set theory," Journal of Mathematical Analysis and Applications, vol. 69, no. 1, pp. 205-230, 1979.

[2] O. Kaleva and S. Seikkala, "On fuzzy metric spaces," Fuzzy Sets and Systems, vol. 12, no. 3, pp. 215-229, 1984.

[3] I. Kramosil and J. Michalek, "Fuzzy metrics and statistical metric spaces," Kybernetika, vol. 11, pp. 336-344, 1975.
[4] O. Kaleva, "A comment on the completion of fuzzy metric spaces," Fuzzy Sets and Systems, vol. 159, no. 16, pp. 2190-2192, 2008.

[5] J.-X. Fang, "An improved completion theorem of fuzzy metric spaces," Fuzzy Sets and Systems, vol. 166, no. 1, pp. 65-74, 2011.

[6] H. Huang and C. Wu, "On the triangle inequalities in fuzzy metric spaces," Information Sciences, vol. 177, no. 4, pp. 1063-1072, 2007.

[7] $\mathrm{H}$. Huang and $\mathrm{C}$. Wu, "On the completion of fuzzy metric spaces," Fuzzy Sets and Systems, vol. 159, no. 19, pp. 2596-2605, 2008.

[8] B.-S. Lee, S.-J. Lee, and K.-M. Park, "The completions of fuzzy metric spaces and fuzzy normed linear spaces," Fuzzy Sets and Systems, vol. 106, no. 3, pp. 469-473, 1999.

[9] F. Shi, "Pointwise pseudo-metrics in-fuzzy set theory $L$-fuzzy set theory," Fuzzy Sets and Systems, vol. 121, no. 2, pp. 209-216, 2001.

[10] J.-Z. Xiao, X.-H. Zhu, and X. Jin, "Fixed point theorems for nonlinear contractions in Kaleva-Seikkala's type fuzzy metric spaces," Fuzzy Sets and Systems, vol. 200, pp. 65-83, 2012.

[11] V. Balopoulos, A. Hatzimichailidis, and B. Papadopoulos, "Distance and similarity measures for fuzzy operators," Information Sciences, vol. 177, no. 11, pp. 2336-2348, 2007.

[12] H. Bustince, E. Barrenechea, and M. Pagola, "Relationship between restricted dissimilarity functions, restricted equivalence functions and normal EN-functions: image thresholding invariant," Pattern Recognition Letters, vol. 29, no. 4, pp. 525-536, 2008.

[13] X. C. Liu, "Entropy, distance measure and similarity measure of fuzzy sets and their relations," Fuzzy Sets and Systems, vol. 52, pp. 305-318, 1992.

[14] J.-L. Fan and Y.-L. Ma, "Some new fuzzy entropy formulas," Fuzzy Sets and Systems, vol. 128, no. 2, pp. 277-284, 2002.

[15] D. Guha and D. Chakraborty, "A new approach to fuzzy distance measure and similarity measure between two generalized fuzzy numbers," Applied Soft Computing, vol. 10, no. 1, pp. 90-99, 2010.

[16] D.-H. Peng, C.-Y. Gao, and Z.-F. Gao, "Generalized hesitant fuzzy synergetic weighted distance measures and their application to multiple criteria decision-making," Applied Mathematical Modelling, vol. 37, no. 8, pp. 5837-5850, 2013.

[17] W. Wang and X. Xin, "Distance measure between intuitionistic fuzzy sets," Pattern Recognition Letters, vol. 26, no. 13, pp. 2063-2069, 2005.

[18] D. Van der Weken, M. Nachtegael, and E. E. Kerre, "Using similarity measures and homogeneity for the comparison of images," Image and Vision Computing, vol. 22, no. 9, pp. 695-702, 2004.

[19] Z. Xu, "A method based on distance measure for intervalvalued intuitionistic fuzzy group decision making," Information Sciences, vol. 180, no. 1, pp. 181-190, 2010.

[20] Z. Xu and M. Xia, "Distance and similarity measures for hesitant fuzzy sets," Information Sciences, vol. 181, no. 11, pp. 2128-2138, 2011. 
[21] H. Zhang, W. Zhang, and C. Mei, "Entropy of interval-valued fuzzy sets based on distance and its relationship with similarity measure," Knowledge-Based Systems, vol. 22, no. 6, pp. 449-454, 2009.

[22] G. Polya, Mathematics and Plausible Reasoning, Vol. I, Induction and Analogy in Mathematics, Princeton University Press, Princeton, NJ, USA, 1954.

[23] G. Wang and H. Zhou, "Quantitative logič," Information Sciences, vol. 179, no. 3, pp. 226-247, 2009.

[24] R. Rajagopalan, "Qualitative reasoning about dynamic change in the spatial properties of a physical system," Doctoral Dissertation, The University of Texas at Austin, Austin, TX, USA, 1995.

[25] D. Dubois and H. Prade, "Operations on fuzzy numbers," International Journal of Systems Science, vol. 9, no. 6, pp. 613-626, 1978.

[26] C. Chakraborty, D. Chakraborty, and Y.L. Yue, "A theoretical development on a fuzzy distance measure for fuzzy numbers," Mathematical and Computer Modelling, vol. 43, no. 3-4, pp. 254-261, 2006.

[27] P. Diamond and P. Kloeden, Metric Spaces of Fuzzy Sets: Theory and Applications, World Scientific, Singapore, 1994.

[28] P. Grzegorzewski, "Metrics and orders in space of fuzzy numbers," Fuzzy Sets and Systems, vol. 97, no. 1, pp. 83-94, 1998.

[29] L. G. Maria, S. Laerte, and S. Luciano, "Option price sensitivities through fuzzy numbers," Computers \& Mathematics with Applications, vol. 61, pp. 515-526, 2011.

[30] C. X. Wu and M. Ma, "Embedding problems of fuzzy number space: Part I," Fuzzy Sets and Systems, vol. 44, pp. 33-38, 1991.

[31] R. Moore and W. Lodwick, "Interval analysis and fuzzy set theory," Fuzzy Sets and Systems, vol. 135, no. 1, pp. 5-9, 2003.

[32] B. Qing Hu, S. Wang, and S. Wang, "A novel approach in uncertain programming part I: new arithmetic and order relation for interval numbers," Journal of Industrial \& Management Optimization, vol. 2, no. 4, pp. 351-371, 2006.

[33] Z. Xu and R. R. Yager, "Some geometric aggregation operators based on intuitionistic fuzzy sets," International Journal of General Systems, vol. 35, no. 4, pp. 417-433, 2006.

[34] J. Chen, S. Li, S. Ma, and X. Wang, " $m$-polar fuzzy sets: an extension of bipolar fuzzy sets," The Scientific World Journal, vol. 2014, Article ID 416530, 8 pages, 2014.

[35] H. Garg, "A new possibility degree measure for intervalvalued $q$-rung orthopair fuzzy sets in decision-making $q$-rung orthopair fuzzy sets in decision-making," International Journal of Intelligent Systems, vol. 36, no. 1, pp. 526-557, 2021.

[36] H. Garg, "CN-q-ROFS: connection number based $q$-rung orthopair fuzzy set and their application to decision-making process," International Journal of Intelligent Systems, vol. 36, no. 7, pp. 1-38, 2021.

[37] H. Garg, Z. Ali, and T. Mahmood, "Generalized dice similarity measures for complex $q$-rung orthopair fuzzy sets and its application," Complex and Intelligent Systems, vol. 7, 2021.

[38] S.-P. Wan, S.-Q. Li, and J.-Y. Dong, "A three-phase method for Pythagorean fuzzy multi-attribute group decision making and application to haze management," Computers \& Industrial Engineering, vol. 123, pp. 348-363, 2018.

[39] S.-P. Wan, Z. Jin, and J.-Y. Dong, "Pythagorean fuzzy mathematical programming method for multi-attribute group decision making with Pythagorean fuzzy truth degrees," Knowledge and Information Systems, vol. 55, no. 2, pp. 437-466, 2018.

[40] S.-P. Wan, Z. Jin, and J.-Y. Dong, "A new order relation for Pythagorean fuzzy numbers and application to multi-attribute group decision making," Knowledge and Information Systems, vol. 62, no. 2, pp. 751-785, 2020.

[41] M. Baczynski and B. Jayaram, "Fuzzy implications," Studies in Fuzziness and Soft Computing, Vol. 231, Springer, Berlin, Germany, 2008.

[42] G.-J. Wang and Y. Leung, "Integrated semantics and logic metric spaces," Fuzzy Sets and Systems, vol. 136, no. 1, pp. 71-91, 2003.

[43] L. Wang, H. Garg, and N. Li, "Pythagorean fuzzy interactive Hamacher power aggregation operators for assessment of express service quality with entropy weight," Soft Computing, vol. 25, pp. 973-993, 2021.

[44] B. E. Rhoades, "A comparison of various definitions of contractive mappings," Transactions of the American Mathematical Society, vol. 226, p. 257, 1977.

[45] P. Wang, P. Zhang, and Z. Li, "A three-way decision method based on Gaussian kernel in a hybrid information system with images: an application in medical diagnosis," Applied Soft Computing, vol. 77, pp. 734-749, 2019.

[46] F. Xiao, "Multi-sensor data fusion based on the belief divergence measure of evidences and the belief entropy," Information Fusion, vol. 46, pp. 23-32, 2019.

[47] K. Rahman, A. Sanan, A. Saleem, and Y. K. Muhammad, "Some induced generalized Einstein aggregating operators and their application to group decision-making problem using intuitionistic fuzzy numbers," Annals of Optimization Theory and Practices, vol. 3, no. 3, pp. 15-49, 2020.

[48] P. A. Ejegwa, I. C. Onyeke, and V. Adah, "An algorithm for an improved intuitionistic fuzzy correlation measure with medical diagnostic application," Annals of Optimization Theory and Practices, vol. 3, no. 3, pp. 51-68, 2020.

[49] M. Hassanpour, "Classification of seven Iranian recycling industries using MCDM models," Annals of Optimization Theory and Practices, vol. 3, no. 4, pp. 37-52, 2020.

[50] P. Talukdar, S. Goala, P. Dutta, and B. Limboo, "Fuzzy multicriteria decision making in medical diagnosis using an advanced distance measure on linguistic Pythagorean fuzzy sets," Annals of Optimization Theory and Practices, vol. 3, no. 4, pp. 113-131, 2020.

[51] J. Ma, M. Atef, A. M. Khalil, N. Hassan, and G.-X. Chen, "Novel models of fuzzy rough coverings based on fuzzy $\alpha$-neighborhood and its application to decision-making $\beta$-neighborhood and its application to decision-making," IEEE Access, vol. 8, pp. 224354-224364, 2020.

[52] J. Yang, S. Li, Z. Xu, H. Liu, and W. Yao, "An understandable way to extend the ordinary linear order on real numbers to a linear order on interval numbers," IEEE Transactions on Fuzzy Systems, p. 1, 2020. 